Homology, Homotopy and Applications, vol.3, No.9, 2001, pp.193-224

\title{
THE COHOMOLOGY RING OF FREE LOOP SPACES
}

\author{
LUC MENICHI
}

(communicated by Lionel Schwartz)

\begin{abstract}
Let $X$ be a simply connected space and $\mathbb{k}$ a commutative ring. Goodwillie, Burghelea and Fiedorowicz proved that the Hochschild cohomology of the singular chains on the space of pointed loops $H H^{*} S_{*}(\Omega X)$ is isomorphic to the free loop space cohomology $H^{*}\left(X^{S^{1}}\right)$. We prove that this isomorphism is compatible with the usual cup product on $H^{*}\left(X^{S^{1}}\right)$ and the cup product of Cartan and Eilenberg on $H H^{*} S_{*}(\Omega X)$. In particular, we make explicit the algebra $H^{*}\left(X^{S^{1}}\right)$ when $X$ is a suspended space, a complex projective space or a finite $\mathrm{CW}$ complex of dimension $p$ such that $\frac{1}{(p-1) !} \in \mathbb{k}$.
\end{abstract}

\section{Introduction}

Let $X^{S^{1}}$ (respectively $\Omega X$ ) be the space of free loops (respectively of pointed loops) of the space $X$. We work over an arbitrary commutative ring $\mathbb{k}$ and for any space $Y$, we denote by $S_{*}(Y)$ the normalized singular chains with coefficients in $\mathbb{k}$.

Recall that the diagonal map $Y \rightarrow Y \times Y$ induces a natural diagonal $S_{*}(Y) \rightarrow$ $S_{*}(Y \times Y) \rightarrow S_{*}(Y) \otimes S_{*}(Y)$ such that $S_{*}(Y)$ is a differential graded coalgebra. The homology of the dual algebra $S^{*}(Y)=\operatorname{Hom}\left(S_{*}(Y), \mathbb{k}\right)$ is the singular cohomology of $Y$. The induced product on $H^{*}(Y)$, called the cup product, makes $H^{*}(Y)$ into a commutative graded algebra.

Now recall from the book of Cartan and Eilenberg [8, XI.6] that, if $A$ denotes an algebra (over $\mathbb{k}$ ) equipped with a diagonal $A \rightarrow A \otimes A$ then the Hochschild cohomology of $A$ (with coefficients in $A^{\vee}=\operatorname{Hom}(A, \mathbb{k})[\mathbf{2 9}, 1.5 .5]$ ), denoted $H H^{*}(A)$, is naturally equipped with a cup product. If $A$ is a Hopf algebra, $H H^{*}(A)$ becomes a graded algebra with this product. These results extend to the case when $A$ is a differential graded algebra. In particular, since $S_{*}(\Omega X)$ is a differential graded Hopf algebra, $H H^{*}\left(S_{*}(\Omega X)\right)$ is naturally a graded algebra. Our main result reads

Research supported by the University of Toronto (NSERC grants RGPIN 8047-98 and OGP000 $7885)$

Received December 12, 2000, revised July 9, 2001; published on July 13, 2001.

2000 Mathematics Subject Classification: 55P35, 16E40, 55P62, 57T30, 55U10.

Key words and phrases: Hochschild homology, free loop space, cup product, bar construction, simplicial space, Hopf algebra up to homotopy, Adams-Hilton model, Sullivan model, Perturbation Lemma.

(c) 2001, Luc Menichi. Permission to copy for private use granted. 
Theorem $\mathbf{A}$ (Theorem 2). If $X$ is a path connected space, the isomorphism of graded modules

$$
H H^{*}\left(S_{*}(\Omega X)\right) \cong H^{*}\left(X^{S^{1}}\right)
$$

established by Goodwillie in [19], Burghelea and Fiedorowicz in [7], preserves cup products.

We emphasize that Theorem A allows us to compute the algebra $H^{*}\left(X^{S^{1}}\right)$ from the cellular structure of $X$ and from a cellular approximation of the diagonal map. Indeed, if we consider the Adams-Hilton model [1] of the space $X$, $\mathcal{A}(X) \stackrel{\simeq}{\longrightarrow} S_{*}(\Omega X)$ and the natural diagonal map

$$
\mathcal{A}(X) \stackrel{\mathcal{A}(\Delta)}{\longrightarrow} \mathcal{A}(X \times X) \longrightarrow \mathcal{A}(X) \otimes \mathcal{A}(X)
$$

as defined in [2, Lemma 8.2], then we prove

Theorem $\mathbf{B}$ (Theorem 11). If $X$ is a simply connected $C W$ complex then the two graded commutative algebras

$$
H H^{*}(\mathcal{A}(X)) \quad \text { and } \quad H^{*}\left(X^{S^{1}}\right)
$$

are isomorphic.

With Theorem B, we are reduced to a purely algebraic problem: computing the algebra $H H^{*}(A)$ when $A$ is a free graded algebra which is also a differential graded Hopf algebra up to homotopy (notion defined page 203). In section 5, using the Perturbation Lemma, we construct a small algebra up to homotopy whose homology is isomorphic to $H H^{*}(A)$.

In section 6 , we make explicit this algebra up to homotopy in the following particular case. Consider a cocommutative differential graded coalgebra $C$. Its dual $C^{\vee}=\operatorname{Hom}(C, \mathbb{k})$ is a commutative differential graded algebra. Recall that for any commutative algebra $A$, its Hochschild homology denoted $H H_{*}(A)$ is endowed with the shuffle product $[8, \mathrm{XI} .6]$. So $H H_{*}\left(C^{\vee}\right)$ is a commutative graded algebra. On the other hand, the cobar construction on $C$, denoted $\Omega C$, is a cocommutative differential graded Hopf algebra [39, $0.6(2)]$. So the cup product of Cartan and Eilenberg is defined on $H H^{*}(\Omega C)$.

Theorem $\mathbf{C}$ (Theorem 18). Let $C$ be a simply connected cocommutative differential graded coalgebra $\mathbb{k}$-free of finite type. The isomorphism of graded modules established by Jones and McCleary in [26, Theorem A] (See also [21, Theorem II] and [38, Theorem 1.1])

$$
H H^{*}(\Omega C) \cong H H_{*}\left(C^{\vee}\right)
$$

is a morphism of commutative graded algebras.

In the last two sections, we give explicit computations of free loop spaces cohomologies. In section 7 we consider the case $X=\Sigma Y$, the suspension of a space $Y$.

Theorem $\mathbf{D}$ (Theorem 27). Let $Y$ be a path connected space such that $H_{*}(Y)$ is $\mathbb{k}$-free of finite type. Then the cup product on $H^{*}(Y)$ determines a product on $H H_{*}\left(H^{*}(\Sigma Y)\right)$ such that $H H_{*}\left(H^{*}(\Sigma Y)\right)$ becomes a commutative graded algebra. This algebra is naturally isomorphic to the algebra $H^{*}\left((\Sigma Y)^{S^{1}}\right)$. 
Notice that if the cup product on $H^{*}(Y)$ is trivial then this product on $H H_{*}\left(H^{*}(\Sigma Y)\right)$ is the shuffle product (Corollary 28). In section 8, we give two other cases where the algebra $H^{*}\left(X^{S^{1}}\right)$ is isomorphic to the Hochschild homology of a commutative differential graded algebra.

First $X=\mathbb{C P}^{n}$, the $n$-dimensional complex projective space or $X=\mathbb{H} \mathbb{P}^{n}$, the $n$-dimensional quaternionic projective space.

Theorem E(Proposition 36). There exists isomorphisms of commutative graded algebras

$$
H^{*}\left(\left(\mathbb{C P}^{n}\right)^{S^{1}}\right) \cong H H_{*}\left(H^{*}\left(\mathbb{C P}^{n}\right)\right)
$$

and

$$
H^{*}\left(\left(\mathbb{H} \mathbb{P}^{n}\right)^{S^{1}}\right) \cong H H_{*}\left(H^{*}\left(\mathbb{H} \mathbb{P}^{n}\right)\right)
$$

Secondly, consider a simply-connected CW-complex $X$ of finite type such that all the integers in the range $[2$, dimension of $X)$ are units in $\mathbb{k}$. When $\mathbb{k}=\mathbb{Q}$, Sullivan constructed a cochain algebra $A_{P L}(X)$, called the polynomial differential forms $[\mathbf{1 8}$, $\S 10]$, and proved with Vigué-Poirrier [41] that there is a natural isomorphism of commutative graded algebras

$$
H^{*}\left(X^{S^{1}}\right) \cong H H_{*}\left(A_{P L}(X)\right) .
$$

Over an arbitrary commutative ring $\mathbb{k}$, Anick $[\mathbf{2}$, Proposition 8.7(a)], extending Sullivan's result, constructed a cochain algebra $\mathcal{C}^{*}(\mathbf{L}(X))$ weakly equivalent as cochain algebras (in the sense of [17, page 832]) to the singular cochains on $X, S^{*}(X)$. We extend the result of Sullivan and Vigué-Poirrier in this new context:

Theorem $\mathbf{F}$ (Theorem 39). For $X$ as above, there is a natural isomorphism of graded commutative algebras

$$
H^{*}\left(X^{S^{1}}\right) \cong H H_{*}\left(\mathcal{C}^{*}(\mathbf{L}(X))\right) .
$$

When $\mathbb{k}$ is a field, this Theorem has been first proved by Dupont and Hess $[\mathbf{1 2}, \mathbf{1 3}$.

We now compare our results to others. Indeed the problem of computing the algebra $H^{*}\left(X^{S^{1}}\right)$ has been considered by many people.

The algebra $H^{*}\left(X^{S^{1}}\right)$ can sometimes be computed via spectral sequences. For example, Kuribayashi and Yamaguchi [27], by solving extensions problems by applications of the Steenrod operations, were able to compute, via the Eilenberg-Moore spectral sequence, the algebra $H^{*}\left(X^{S^{1}}\right)$ for some simple spaces. Using fibrewise homotopy technics [10], Crabb and James have, before us, computed the cohomology with integer coefficients $H^{*}\left(\left(\mathbb{C P}^{n}\right)^{S^{1}} ; \mathbb{Z}\right)$. In $[\mathbf{9}]$, Cohen gives a combinatorial model for the free loop space on a suspension, $(\Sigma Y)^{S^{1}}$. It should be interesting to see if one can deduce from this model, the algebra structure or the Steenrod operations on $H^{*}\left((\Sigma Y)^{S^{1}}\right)$.

Another approach is to use algebraic models like us. Suppose that $\mathbb{k}$ is a field. Given an Adams-Hilton model of $X, \mathcal{A}(X)$, equipped with its diagonal, Dupont and Hess [14] have constructed, with some indeterminacy, a cochain complex equipped 
with a product whose homology is isomorphic to the algebra $H^{*}\left(X^{S^{1}}\right)$. They don't mention any relation between their complex and the Hochschild complex on $\mathcal{A}(X)$. Again, when $\mathbb{k}$ is a field, Ndombol and Thomas [35] have found an "Eckmann-Hilton dual" to our main result (Theorem A): using the "strongly homotopy commutative" structure on $S^{*}(X)$, they constructed a product on the Hochschild homology of $S^{*}(X)$ and proved that the isomorphism of Jones [25] $H^{*}\left(X^{S^{1}}\right) \cong H H_{*}\left(S^{*}(X)\right)$ is an isomorphism of algebras. The equivalence between their result and our main result has by now been proved by Idrissi $[\mathbf{2 4}]$.

We thank S. Halperin, J.-C. Thomas and M. Vigué for their constant support. The main results of this paper were expound in September 1999 at the GDR Topologie algébrique meeting in Paris Nord.

\section{Algebraic preliminaries and notation}

We work over a commutative ring $\mathbb{k}$. We denote by $p^{\mathbb{k}}$ and $\frac{\mathbb{k}}{p \mathbb{k}}$ respectively the kernel and cokernel of the multiplication by $p$ in $\mathbb{k}$.

DGA stands for differential graded algebra, DGC for differential graded coalgebra, DGH for differential graded Hopf algebra and CDGA for commutative DGA. The denomination "chain" will be restricted to objects with a non-negative lower degree and "cochain" to those with a non-negative upper degree.

The degree of an element $x$ is denoted $|x|$. The suspension of a graded module $V$ is the graded module $s V$ such that $(s V)_{i+1}=V_{i}$. Let $C$ be an augmented complex. The kernel of the augmentation is denoted $\bar{C}$.

The exterior algebra on an element $v$ is denoted $E v$. The free divided powers algebra on an element $v$, denoted $\Gamma v$, is

- the free graded algebra generated by $\gamma^{i}(v), i \in \mathbb{N}^{*}$, divided by the relations $\gamma^{i}(v) \gamma^{j}(v)=\frac{(i+j) !}{i ! j !} \gamma^{i+j}(v)$, if $|v|$ is even,

- and is just $E v$ when $|v|$ is odd.

The tensor algebra on a graded module $V$ is denoted TA $V$. The tensor coalgebra is denoted TCV. Their common underlying module is simply denoted $T V$. Given a conilpotent coalgebra $C$ then any morphism $\varphi: \bar{C} \rightarrow V$ lifts uniquely to a unique morphism $\Psi: C \rightarrow \mathrm{TC} V$ of coaugmented coalgebras. The formula for $\Psi$ is given by

$$
\Psi(c)=\sum_{i=1}^{+\infty} \varphi^{\otimes i} \circ \bar{\Delta}_{C}^{\otimes i-1}(c), c \in \bar{C}
$$

where $\Delta_{C}^{i-1}: \bar{C} \rightarrow \bar{C}^{\otimes i}$ is the iterated reduced diagonal of $C$.

Let $A$ be an augmented DGA. The bar resolution of $A$, denoted $\mathrm{B}(A ; A ; A)$, is the unique $(A, A)$-bimodule $\left(A \otimes \mathrm{T}(s \bar{A}) \otimes A, d_{1}+d_{2}\right)$ such that $d_{1}$ denote the differential 
obtained by tensorization and such that, for any $\left[s a_{1}|\cdots| s a_{k}\right] \in \mathrm{T}(s \bar{A})$

$$
\begin{aligned}
d_{2}\left[s a_{1}|\cdots| s a_{k}\right]= & a_{1}\left[s a_{2}|\cdots| s a_{k}\right] \\
& +\sum_{i=1}^{k-1}(-1)^{\left|s a_{1}\right|+\cdots+\left|s a_{i}\right|}\left[s a_{1}|\cdots| s a_{i} a_{i+1}|\cdots| s a_{k}\right] \\
& -(-1)^{\left|s a_{1}\right|+\cdots+\left|s a_{k-1}\right|}\left[s a_{1}|\cdots| s a_{k-1}\right] a_{k} .
\end{aligned}
$$

The (reduced) bar construction on $A$, denoted $\mathrm{B}(A)$, is the coaugmented DGC (TCs $\bar{A}, d_{1}+d_{2}$ ) whose underlying complex $\left(\mathrm{T} s \bar{A}, d_{1}+d_{2}\right)$ coincides with $\mathbb{k} \otimes_{A}$ $\mathrm{B}(A ; A ; A) \otimes_{A} \mathbb{k}[\mathbf{1 7}, \S 4]$. The cyclic bar construction or Hochschild complex is the complex $A \otimes_{A \otimes A^{o p}} \mathrm{~B}(A ; A ; A)$ denoted $\mathrm{C}(A)$. Explicitly $\mathrm{C}(A)$ is the complex $(A \otimes$ $\left.\mathrm{T}(s \bar{A}), d_{1}+d_{2}\right)$ with $d_{1}$ obtained by tensorization and

$$
\begin{aligned}
d_{2} a\left[s a_{1}|\cdots| s a_{k}\right]= & (-1)^{|a|} a a_{1}\left[s a_{2}|\cdots| s a_{k}\right] \\
& +\sum_{i=1}^{k-1}(-1)^{\varepsilon_{i}} a\left[s a_{1}|\cdots| s a_{i} a_{i+1}|\cdots| s a_{k}\right] \\
& -(-1)^{\left|s a_{k}\right| \varepsilon_{k-1}} a_{k} a\left[s a_{1}|\cdots| s a_{k-1}\right] ;
\end{aligned}
$$

The Hochschild homology is the homology of the cyclic bar construction:

$$
H H_{*}(A):=H_{*}(\mathrm{C}(A)) .
$$

The Hochschild cohomology is the graded module

$$
H H^{*}(A):=H^{*}\left(\operatorname{Hom}_{(A, A)}\left(\mathrm{B}(A ; A ; A), A^{\vee}\right)\right)=H^{*}\left(\mathrm{C}(A)^{\vee}\right)
$$

where $A^{\vee}$ is considered as an $(A, A)$-bimodule.

Let $A$ and $B$ be two augmented DGA's. The Alexander-Whitney map is the unique morphism of $(A \otimes B, A \otimes B)$-bimodules

$$
A W: \mathrm{B}(A \otimes B ; A \otimes B ; A \otimes B) \rightarrow \mathrm{B}(A ; A ; A) \otimes \mathrm{B}(B ; B ; B)
$$

such that the image of a typical element $\left[s\left(a_{1} \otimes b_{1}\right)|\cdots| s\left(a_{k} \otimes b_{k}\right)\right]$ is

$$
\sum_{i=0}^{k}(-1)^{\zeta_{i}}\left[s a_{1}|\cdots| s a_{i}\right] a_{i+1} \cdots a_{k} \otimes b_{1} \cdots b_{i}\left[s b_{i+1}|\cdots| s b_{k}\right] .
$$

Here $[\mathbf{3 2}, 3.7]$

$$
\zeta_{i}=\sum_{1 \leqslant l<j \leqslant k}\left|b_{l}\right|\left|a_{j}\right|+\sum_{j=i+1}^{k}(j-i)\left|a_{j}\right|+\sum_{j=1}^{i-1}(i-j)\left|b_{j}\right| .
$$

$A W$ is natural and associative exactly. It is also commutative up to a homotopy of $(A \otimes B, A \otimes B)$-bimodules. So we get an Alexander-Whitney map for the cyclic bar construction

$$
A W: \mathrm{C}(A \otimes B) \rightarrow \mathrm{C}(A) \otimes \mathrm{C}(B) .
$$

Consider an augmented DGA $K$ equipped with a morphism of augmented DGA's 
$\Delta: K \rightarrow K \otimes K$. Then the composite

$$
\Delta: \mathrm{C}(K) \stackrel{\mathrm{C}(\Delta)}{\longrightarrow} \mathrm{C}(K \otimes K) \stackrel{A W}{\longrightarrow} \mathrm{C}(K) \otimes \mathrm{C}(K)
$$

is a morphism of augmented complex. Therefore $H H^{*}(K)$ has a product. This is the cup product of Cartan and Eilenberg [8, XI.6], also called Hopf wedge product by Mac Lane [30, VIII.4.(4.5)]. In particular, if $K$ is a DGH then $\mathrm{C}(K)$ is a DGC and $H H^{*}(K)$ is a graded algebra.

\section{From the chains on the based loops to the chains on the free loops}

The object of this section is to prove the following theorem linking the chains on the based loops of a space to the chains on its free loops.

Theorem 2. Let $X$ be a path connected pointed space. Then there is a natural DGC quasi-isomorphism

$$
\mathrm{C}\left(S_{*}(\Omega X)\right) \stackrel{\simeq}{\rightarrow} S_{*}\left(X^{S^{1}}\right) .
$$

In particular, $H H^{*} S_{*}(\Omega X) \cong H^{*}\left(X^{S^{1}}\right)$ as graded algebras.

Goodwillie [19], Burghelea and Fiedorowicz [7] proved the isomorphism $H H^{*} S_{*}(\Omega X) \cong H^{*}\left(X^{S^{1}}\right)$ as graded modules only. To obtain our theorem, we will follow their proofs. We introduce first some terminology about simplicial objects.

Let $\mathcal{C}$ be a category. A simplicial $\mathcal{C}$-object $X$ is a non-negative graded object together with morphisms $d_{i}: X_{n} \rightarrow X_{n-1}$ and $s_{i}: X_{n} \rightarrow X_{n+1}, 0 \leqslant i \leqslant n$ satisfying some well-known relations [30, VIII.5.2]. A cosimplicial $\mathcal{C}$-object is a non-negative graded object together with morphisms $\delta_{i}: X^{n-1} \rightarrow X^{n}$ and $\sigma_{i}: X^{n+1} \rightarrow X^{n}$, $0 \leqslant i \leqslant n$ satisfying the opposite relations $[\mathbf{6}, \mathrm{X} .2 .1(\mathrm{i})]$. If $\mathcal{C}$ is a category equipped with a tensor product $\otimes$ (more precisely a monoidal category [31, VII.1]) then the tensor product of two simplicial $\mathcal{C}$-objects $X=\left(X_{n}, d_{i}, s_{i}\right)$ and $Y=\left(Y_{n}, d_{i}, s_{i}\right)$ is the simplicial $\mathcal{C}$-object $X \otimes Y=\left(X_{n} \otimes Y_{n}, d_{i} \otimes d_{i}, s_{i} \otimes s_{i}\right)$.

Consider $\mathcal{C}$ to be the category of complexes. To any simplicial $\mathcal{C}$-object (i.e. simplicial complex) $X$, we can associate a complex in the category $\mathcal{C}$ (i.e. a complex of complexes) denoted $K_{N}(X)$ known as the normalized chain complex of $X[\mathbf{3 0}$, VIII.6 for the category of modules]. Consider two simplicial complexes $A$ and $B$. We have an Alexander-Whitney morphism of complexes of complexes [30, VIII.8.6] $A W: K_{N}(A \otimes B) \rightarrow K_{N}(A) \otimes K_{N}(B)$. Every complex of complexes can be condensated [30, X.9.1] into a single complex. So by composing the functor $K_{N}$ and the condensation functor, we have a functor, called the realization and denoted $\mid$ from the category of simplicial complexes to the category of complexes, equipped with an Alexander-Whitney morphism of complexes $A W:|A \otimes B| \rightarrow|A| \otimes|B|$ for any simplicial complexes $A$ and $B$. In particular, | | induces a functor from the category of simplicial DGC's to the category of DGC's (Recall that a simplicial DGC can be defined either as a simplicial object in the category of DGC's or as a coalgebra in the category of simplicial complexes.). 
Given any two topological spaces $X$ and $Y$, the caligraphic notations

$$
\mathcal{A W}: S_{*}(X \times Y) \rightarrow S_{*}(X) \otimes S_{*}(Y) \text { and } \mathcal{E} \mathcal{Z}: S_{*}(X) \otimes S_{*}(Y) \rightarrow S_{*}(X \times Y)
$$

are reserved for the standard normalized Alexander-Whitney map and to the standard normalized Eilenberg-Zilber map concerning singular chains [11, VI.12.27-8].

Example 3. The cyclic bar construction for differential graded algebras. Let $A$ be a DGA. Then there is a simplicial complex $\Gamma A$ defined by $\Gamma_{n} A=A \otimes \cdots \otimes A=A^{\otimes n+1}$,

$$
\begin{aligned}
d_{0} a\left[a_{1}|\cdots| a_{n}\right] & =a a_{1}\left[a_{2}|\cdots| a_{n}\right], \\
d_{i} a\left[a_{1}|\cdots| a_{n}\right] & =a\left[a_{1}|\cdots| a_{i} a_{i+1}|\cdots| a_{n}\right] \text { for } 1 \leqslant i \leqslant n-1, \\
d_{n} a\left[a_{1}|\cdots| a_{n}\right] & =a_{n} a\left[a_{1}|\cdots| a_{n-1}\right], \\
s_{i} a\left[a_{1}|\cdots| a_{n}\right] & =a\left[a_{1}|\cdots| a_{i}|1| a_{i+1}|\cdots| a_{n}\right] \text { for } 0 \leqslant i \leqslant n .
\end{aligned}
$$

The complex $|\Gamma A|$ is exactly (signs included) $\mathrm{C}(A)$ the cyclic bar construction of $A$. If $K$ is a DGH then $\Gamma K$ with the diagonal

$$
\Gamma K \stackrel{\Gamma\left(\Delta_{K}\right)}{\longrightarrow} \Gamma(K \otimes K) \cong \Gamma K \otimes \Gamma K
$$

is a simplicial DGC and $|\Gamma K|$ is the $\mathrm{DGC} \mathrm{C}(K)$ denoted in section 2.

Example 4. Let $G$ be a topological monoid. The cyclic bar construction of $G[\mathbf{2 9}$, 7.3.10] is the simplicial space $\Gamma G$ defined by $\Gamma_{n} G=G \times \cdots \times G=G^{n+1}$ and with the same formulas for $d_{i}$ and $s_{i}$ as in the cyclic bar construction for DGA's. Since the normalized singular chain functor $S_{*}$ is a functor from topological spaces to DGC's, $S_{*}(\Gamma G)$ is a simplicial DGC. Therefore $\left|S_{*}(\Gamma G)\right|$ is a DGC.

The following Lemma compares the DGC's given by the previous two examples.

Lemma 5. (Compare [19, V.1.2]) Let $G$ be a topological monoid. Then there is a natural DGC quasi-isomorphism $\left|\Gamma S_{*}(G)\right| \stackrel{\simeq}{\rightrightarrows}\left|S_{*}(\Gamma G)\right|$.

Proof. The Eilenberg-Zilber map $\mathcal{E Z}: S_{*}(G)^{\otimes n+1} \rightarrow S_{*}\left(G^{n+1}\right)$ is a DGC quasiisomorphism and therefore defines a morphism of simplicial DGC's $\Gamma S_{*}(G) \rightarrow$ $S_{*}(\Gamma G)$. So, applying the functor | |, we get a DGC quasi-isomorphism.

Let $\Delta^{n}$ be the standard geometric simplex of dimension $n$. Let $\delta_{i}: \Delta^{n-1} \rightarrow \Delta^{n}$ and $\sigma_{i}: \Delta^{n+1} \rightarrow \Delta^{n}$ be the $i$-th face inclusion and the $i$-th degeneracy of $\Delta^{n}$. Then $\gtreqless=\left(\Delta^{n}, \delta_{i}, \sigma_{i}\right)$ is a cosimplicial space $[\mathbf{6}, \mathrm{X} .2 .2(\mathrm{i})]$. The geometric realization $[\mathbf{3 3}$, 11.1] of a simplicial space $X$ is defined as

$$
|X|=\left(\coprod_{n \in \mathbb{N}} X_{n} \times \Delta^{n}\right) / \sim
$$

where $\sim$ is the equivalence relation generated by

$$
\begin{aligned}
\left(d_{i} x, y\right) & \sim\left(x, \delta_{i} y\right), x \in X_{n}, y \in \Delta^{n-1} \\
\text { and } \quad\left(s_{i} x, y\right) & \sim\left(x, \sigma_{i} y\right), x \in X_{n}, y \in \Delta^{n+1} .
\end{aligned}
$$

Recall that $\left|S_{*}(X)\right|$ is a DGC whose diagonal is the composite

$$
\left|S_{*}(X)\right| \stackrel{\left|S_{*}(\Delta)\right|}{\longrightarrow}\left|S_{*}(X \times X)\right| \stackrel{|\mathcal{A \mathcal { W }}|}{\longrightarrow}\left|S_{*}(X) \otimes S_{*}(X)\right| \stackrel{A W}{\longrightarrow}\left|S_{*}(X)\right| \otimes\left|S_{*}(X)\right|
$$


Lemma 6. (Compare [5, Theorem 4.1] and [18, 17(a)]) Let $X$ be a simplicial space, good in the sense of [37, A.4]. Then there is a natural DGC quasi-isomorphism $f:\left|S_{*}(X)\right| \stackrel{\simeq}{\rightarrow} S_{*}(|X|)$.

Proof. Let $\pi_{n}: X_{n} \times \Delta^{n} \rightarrow|X|$ be the quotient map. The morphism $f$ is defined as the composite

$$
S_{i}\left(X_{n}\right) \stackrel{i d_{S_{i}\left(X_{n}\right)} \otimes \kappa_{n}}{\longrightarrow} S_{i}\left(X_{n}\right) \otimes S_{n}\left(\Delta^{n}\right) \stackrel{\mathcal{E Z}}{\longrightarrow} S_{i+n}\left(X_{n} \times \Delta^{n}\right) \stackrel{S_{i+n}\left(\pi_{n}\right)}{\longrightarrow} S_{n+i}(|X|)
$$

where $\kappa_{n} \in S_{n}\left(\Delta^{n}\right)$ is the singular simplex $i d_{\Delta^{n}}$. By a Theorem of Moore $[\mathbf{5}$, Theorem 4.1], $f$ is a quasi-isomorphism of chains complexes. The diagonal map of $|X|$ is equal to the composite

$$
|X| \stackrel{\left|\Delta_{X}\right|}{\longrightarrow}|X \times X| \stackrel{\left(\left|\operatorname{proj}_{1}\right|, \mid \text { proj }_{2} \mid\right)}{\longrightarrow}|X| \times|X|
$$

where $\Delta_{X}$ is the simplicial diagonal of $X$ and where $\operatorname{proj}_{1}$ and $\operatorname{proj}_{2}$ are the simplicial projections on each factors. To check that $f$ is a DGC morphism, we have to show:

$$
(f \otimes f) \circ A W \circ|\mathcal{A} \mathcal{W}| \circ\left|S_{*}\left(\Delta_{X}\right)\right|=\mathcal{A} \mathcal{W} \circ S_{*}\left(\left(\left|\operatorname{proj}_{1}\right|,\left|\operatorname{proj}_{2}\right|\right)\right) \circ S_{*}\left(\left|\Delta_{X}\right|\right) \circ f .
$$

By naturality of $f$, it suffices to show that $f$ behaves well with respect to products of simplicial spaces.

Let $X$ and $Y$ be two simplicial spaces. Then $S_{*}(X)$ and $S_{*}(Y)$ are two simplicial complexes. So we have an Alexander-Whitney map

$$
\left|S_{*}(X) \otimes S_{*}(Y)\right| \stackrel{A W}{\longrightarrow}\left|S_{*}(X)\right| \otimes\left|S_{*}(Y)\right| .
$$

Its formula is given by

$$
\left[\sum_{p+q=n} S_{*}\left(\tilde{d}^{q}\right) \otimes S_{*}\left(d_{0}^{p}\right)\right]: S_{*}\left(X_{n}\right) \otimes S_{*}\left(Y_{n}\right) \longrightarrow \bigoplus_{p+q=n} S_{*}\left(X_{p}\right) \otimes S_{*}\left(Y_{q}\right)
$$

where $\tilde{d}^{q}: X_{n} \rightarrow X_{p}$ is the composite $d_{p+1} \circ \cdots \circ d_{n}(\tilde{d}$ denotes the "last" face operator) and $d_{0}^{p}: Y_{n} \rightarrow Y_{q}$ is the iterated composite of $d_{0}$. In the diagram page 201, there was not enough space for sums $\sum$ and direct sums $\bigoplus$. So we use the indices $p$ and $q$ with the convention $p+q=n$ and the indices $j$ and $k$ with the conventions that $j+k=i$. We use also the maps

$$
\tilde{\delta}^{q}=\delta_{n} \circ \cdots \circ \delta_{p+1}: \Delta^{p} \rightarrow \Delta^{n} \quad \text { and } \quad \delta_{0}^{p}: \Delta^{q} \rightarrow \Delta^{n} .
$$

Both the interchange of factors of a tensor product of modules and of a product of spaces are denoted by $\tau$.

Consider the diagram page 201. 


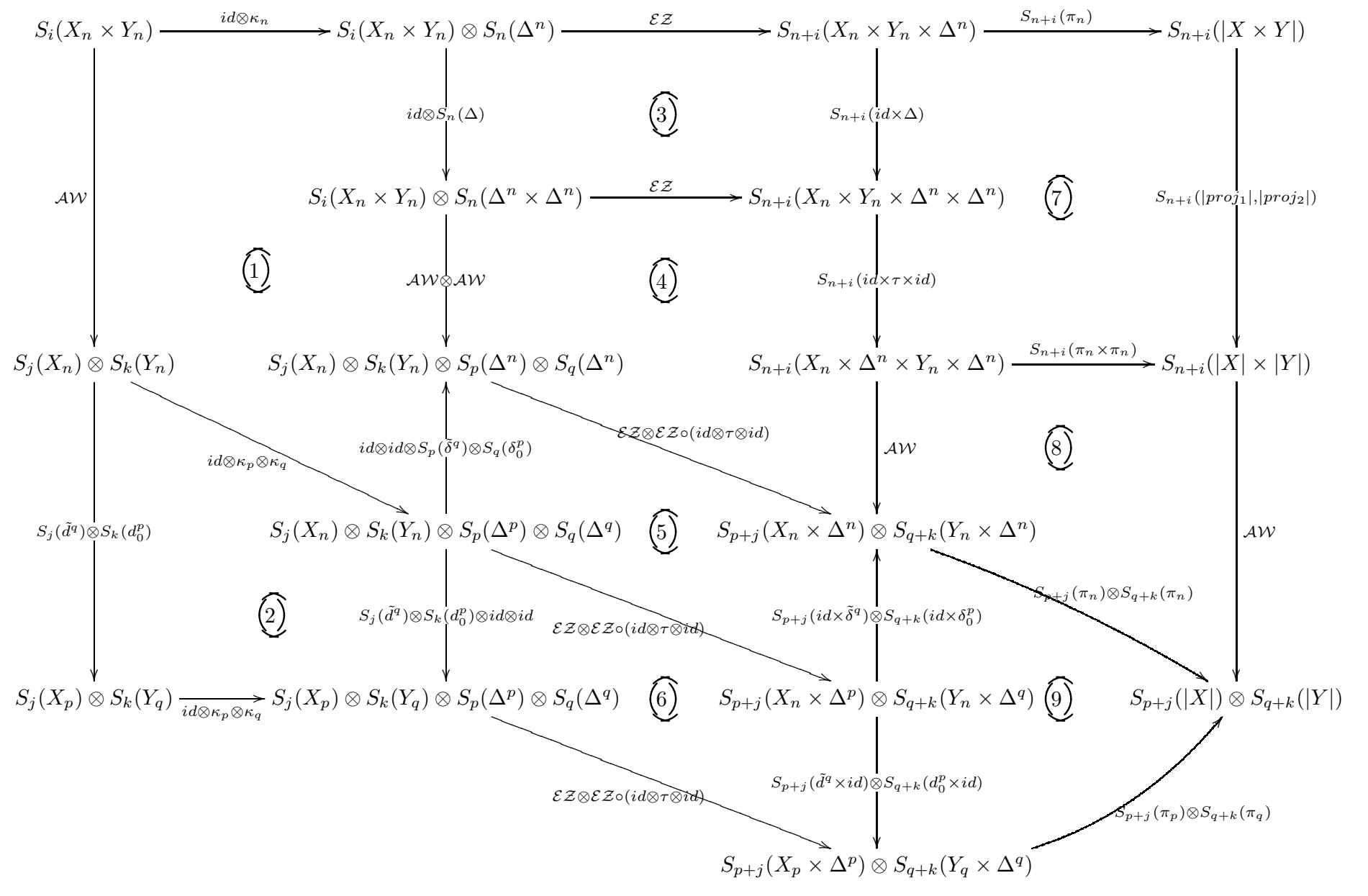


Let's check the commutativity of each subdiagram involved in it.

- 1 commutes since

$$
\left(\mathcal{A} \mathcal{W} \circ S_{n}(\Delta)\right)\left(\kappa_{n}\right)=\sum_{p+q=n}\left(\tilde{\delta}^{q} \otimes \delta_{0}^{p}\right)=\sum_{p+q=n}\left(S_{p}\left(\tilde{\delta}^{q}\right) \otimes S_{q}\left(\delta_{0}^{p}\right)\right)\left(\kappa_{p} \otimes \kappa_{q}\right)
$$

- 2 commutes obviously.

- 3 commutes by naturality of $\mathcal{E Z}$.

- 4 commutes by compatibility of $\mathcal{E Z}$ and $\mathcal{A W}$ [18, I.4.b)].

- 5 and 6 commute by naturality of $(\mathcal{E Z} \otimes \mathcal{E Z}) \circ(i d \otimes \tau \otimes i d)$.

- 7 commutes since

$$
\left(\left|\operatorname{proj}_{1}\right|,\left|\operatorname{proj}_{2}\right|\right) \circ \pi_{n}=\left(\pi_{n} \times \pi_{n}\right) \circ(i d \times \tau \times i d) \circ(i d \times \Delta) .
$$

- 8 commutes by naturality of $\mathcal{A W}$.

- By definition of the equivalence relation $\sim$ defining the geometric realisation of a simplicial space,

$$
\pi_{p} \circ\left(\tilde{d}^{q} \times i d_{\Delta^{p}}\right)=\pi_{n} \circ\left(i d_{X_{n}} \times \tilde{\delta}^{q}\right) \text { and } \pi_{q} \circ\left(d_{0}^{p} \times i d_{\Delta^{q}}\right)=\pi_{n} \circ\left(i d_{Y_{n}} \times \delta_{0}^{q}\right) .
$$

So 9 commutes.

Finally, we have

$$
(f \otimes f) \circ\left[\sum_{p, q} S_{*}\left(\tilde{d}^{q}\right) \otimes S_{*}\left(d_{0}^{p}\right)\right] \circ|\mathcal{A} \mathcal{W}|=\mathcal{A} \mathcal{W} \circ S_{*}\left(\left(\left|\operatorname{proj}_{1}\right|,\left|\operatorname{proj}_{2}\right|\right)\right) \circ f .
$$

Lemma 7. [29, 7.3.15] Let $X$ be a path connected pointed space. Then there is a natural homotopy equivalence $|\Gamma \Omega X| \stackrel{\simeq}{\rightarrow} X^{S^{1}}$.

Proof of Theorem 2. Applying Lemma 5 to the Moore loop space $\Omega X$, Lemma 6 to $\Gamma \Omega X$ and Lemma 7, we obtain the sequence of DGC quasi-isomorphisms:

$$
\mathrm{C} S_{*}(\Omega X)=\left|\Gamma S_{*}(\Omega X)\right| \stackrel{\simeq}{\rightrightarrows}\left|S_{*}(\Gamma \Omega X)\right| \stackrel{\simeq}{\rightarrow} S_{*}(|\Gamma \Omega X|) \stackrel{\simeq}{\rightarrow} S_{*}\left(X^{S^{1}}\right) .
$$

\section{HAH models}

In order to compute the algebra structure of $H H^{*} S_{*}(\Omega X)$, it is necessary to replace $S_{*}(\Omega X)$ by a smaller Hopf algebra. Let's first remark that the cyclic bar construction preserves quasi-isomorphisms.

Property 8. [29, 5.3.5](Compare [17, 4.3(iii)]) Let $f: A \rightarrow B$ be a quasi-isomorphism of augmented DGA's. If $A$ and $B$ are $\mathbb{k}$-semifree then $\mathrm{C}(f): \mathrm{C}(A) \stackrel{\simeq}{\rightarrow} \mathrm{C}(B)$ is a quasi-isomorphism of complexes.

Let $f, g: A \rightarrow B$ be two morphisms of augmented DGA's. A derivation homotopy from $f$ to $g$ is a morphism of graded modules of degree $+1, h: A \rightarrow \bar{B}$ such that 
$d \circ h+h \circ d=f-g$ and $h(x y)=h(x) g(y)+(-1)^{|x|} f(x) h(y)$ for $x, y \in A$. A derivation homotopy from $f$ to $g$ is denoted by $h: f \approx g$. We say that $f$ and $g$ are homotopic if there is a derivation homotopy between them.

Lemma 9. Let $f, g: A \rightarrow B$ be two morphisms of augmented chain algebras. Suppose that $A$ is $\mathbb{k}$-free. If $f$ and $g$ are homotopic then the morphisms of chain complexes $\mathrm{C}(f), \mathrm{C}(g): \mathrm{C}(A) \rightarrow \mathrm{C}(B)$ are chain homotopic.

Proof. We first prove the lemma assuming that $A$ is a chain algebra of the form $(\mathrm{TA} V, \partial)$ where $V$ is a $\mathbb{k}$-free graded module. Consider the Baues-Lemaire cylinder of $A$. This is an augmented chain algebra $I A$ equipped with a commutative diagram of augmented chain algebras

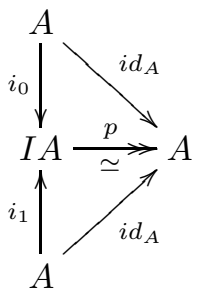

The derivation homotopy between $f$ and $g$ correspond to an morphism of augmented chain algebra $H: I A \rightarrow B$ such that $H \circ i_{0}=f$ and $H \circ i_{1}=g$ ([4, I.7.12] or $[\mathbf{1 7}, 3.5])$. Since $p: I A \stackrel{\simeq}{\rightarrow} A$ is a quasi-isomorphism, by Property $8, \mathrm{C}(p)$ is a quasi-isomorphism. Since $\mathrm{C}(p) \circ \mathrm{C}\left(i_{0}\right)=\mathrm{C}(p) \circ \mathrm{C}\left(i_{1}\right)$ and $C(A)$ is a $\mathbb{k}$-free chain complex, $\mathrm{C}\left(i_{0}\right)$ and $\mathrm{C}\left(i_{1}\right)$ are chain homotopic. Therefore $\mathrm{C}(f)=\mathrm{C}(H) \circ \mathrm{C}\left(i_{0}\right)$ and $\mathrm{C}(g)=\mathrm{C}(H) \circ \mathrm{C}\left(i_{1}\right)$ are chain homotopic.

If $A$ is not a chain algebra of the form (TA $V, \partial$ ), consider a quasi-isomorphism of augmented chain algebras $\Theta:(\mathrm{TA} V, \partial) \stackrel{\simeq}{\rightarrow} A$ where $V$ is $\mathbb{k}$-free. Since there is a derivation homotopy between $\Theta \circ f$ and $\Theta \circ g, \mathrm{C}(\Theta) \circ \mathrm{C}(f)$ and $\mathrm{C}(\Theta) \circ \mathrm{C}(f)$ are chain homotopic. If $A$ is $\mathbb{k}$-free, $\mathrm{C}(\Theta): \mathrm{C}(\mathrm{TA} V, \partial) \stackrel{\simeq}{\rightarrow} C(A)$ is a quasi-isomorphism between $\mathbb{k}$-free chain complexes and so is a chain homotopy equivalence. Therefore $\mathrm{C}(f)$ and $\mathrm{C}(g)$ are chain homotopic.

A Hopf algebra up to homotopy, or HAH, is a DGA $K$ equipped with two morphisms of DGA's $\Delta: K \rightarrow K \otimes K$ and $\varepsilon: K \rightarrow \mathbb{k}$ such that $\left(\varepsilon \otimes i d_{K}\right) \circ \Delta=i d_{K}=$ $\left(i d_{K} \otimes \varepsilon\right) \circ \Delta$ (counitary exactly), $(\Delta \otimes 1) \circ \Delta \approx(1 \otimes \Delta) \circ \Delta$ (coassociative up to homotopy) and $\tau \circ \Delta \approx \Delta$ (cocommutative up to homotopy).

Let $K, K^{\prime}$ be two HAH's. A morphism of augmented DGA's $f: K \rightarrow K^{\prime}$ is a $H A H$ morphism if $\Delta f \approx(f \otimes f) \Delta$ ( $f$ commutes with the diagonals up to homotopy).

Definition 10. Let $X$ be a pointed topological space. A $H A H$ model for $X$ is a chain algebra of the form (TA $V, \partial$ ), where the graded module $V$ is $\mathbb{k}$-free, equipped with a structure of Hopf algebras up to homotopy and with a HAH quasi-isomorphism $\Theta:(\mathrm{TA} V, \partial) \stackrel{\simeq}{\rightarrow} S_{*}(\Omega X)$.

Suppose that $X$ is a simply-connected CW-complex. Its Adams-Hilton model 
$\mathcal{A}(X)$ equipped with its diagonal

$$
\mathcal{A}(X) \stackrel{\mathcal{A}(\Delta)}{\longrightarrow} \mathcal{A}(X \times X) \longrightarrow \mathcal{A}(X) \otimes \mathcal{A}(X) .
$$

is a HAH model for $X[\mathbf{2}$, Proposition 8.3].

Theorem 11. Let $X$ be a path connected pointed space. Let (TAV, $\partial)$ be a HAH model for $X$. There is a isomorphism of graded algebras

$$
H H^{*}(\mathrm{TA} V, \partial) \cong H^{*}\left(X^{S^{1}}\right) \text {. }
$$

Proof. Let $\Theta:(\mathrm{TA} V, \partial) \stackrel{\cong}{\rightrightarrows} S_{*}(\Omega X)$ denote the HAH quasi-isomorphism. By Property $8, \mathrm{C}(\Theta)$ is a quasi-isomorphism. According Lemma 9,

$$
\mathrm{C}\left((\Theta \otimes \Theta) \circ \Delta_{\mathrm{TA} V}\right) \approx \mathrm{C}\left(\Delta_{S_{*}(\Omega X)} \circ \Theta\right) .
$$

By composing with $A W$ and by applying Theorem 2, the quasi-isomorphisms of chain complexes

$$
\mathrm{C}(\mathrm{TA} V, \partial) \stackrel{\mathrm{C}(\Theta)}{\longrightarrow} \mathrm{C} S_{*}(\Omega X) \stackrel{\sim}{\rightrightarrows} S_{*}\left(X^{S^{1}}\right)
$$

commutes with the diagonals up to chain homotopy.

\section{A smaller coalgebra than the cyclic bar construction}

The goal of this section is to replace the huge algebra up to homotopy $\mathrm{C}(\mathrm{TA} V, \partial)^{\vee}$ by a smaller one in order to be able to compute the algebra $H H^{*}(\mathrm{TA} V, \partial)$. When $\mathbb{k}$ is a field, Micheline Vigué in [40] gives a small complex $((\mathbb{k} \oplus s V) \otimes \mathrm{T} V, \delta)$ whose homology is the vector space $H H_{*}(\mathrm{TA} V, \partial)$. In fact, in this section, we show that over any commutative ring $\mathbb{k}$, the complex $((\mathbb{k} \oplus s V) \otimes \mathrm{T} V, \delta)$ is a strong deformation retract of $\mathrm{C}(\mathrm{TA} V, \partial)$.

Definition 12. Let $(Y, d)$ be a complex. A complex $(X, \partial)$ is a strong deformation retract of $(Y, d)$ if there exist two morphisms of complexes $\nabla:(X, \partial) \hookrightarrow(Y, d)$, $f:(Y, d) \rightarrow(X, \partial)$ and a chain homotopy $\Phi:(Y, d) \rightarrow(Y, d)$ such that $f \nabla=i d_{X}$ and $\nabla f-i d_{Y}=d \Phi+\Phi d$. The map $f$ is called the projection and the map $\nabla$ is called the inclusion.

We first consider the case where the differential $\partial$ on $(\mathrm{TA} V, \partial)$ is just obtained by tensorization of the differential of a complex $V$ and is so therefore homogeneous by wordlength.

Consider the tensor algebra TA $V$ on a complex $V$. Define the augmentation on $\mathrm{TA} V$ such that the augmentation ideal $\overline{\mathrm{TA} V}$ is

$$
\mathrm{T}^{+} V=\oplus_{i \geqslant 1} V^{\otimes i}
$$

The bar resolution $\mathrm{B}(\mathrm{TA} V ; \mathrm{TA} V ; \mathrm{TA} V)$ contains a subcomplex $(\mathrm{T} V \otimes(\mathbb{k} \oplus s V) \otimes$ $\left.\mathrm{T} V, d_{1}+d_{2}\right)$, since

$$
d_{2}(a \otimes s v \otimes b)=(-1)^{|a|}(a v \otimes b-a \otimes v b) .
$$


Proposition 13. [29, Proposition 3.1.2] The (TAV, TAV)-bimodule (TV $\otimes$ $\left.(\mathbb{k} \oplus s V) \otimes \mathrm{T} V, d_{1}+d_{2}\right)$ is a strong deformation retract of the bar resolution $\mathrm{B}(\mathrm{TA} V ; \mathrm{TA} V ; \mathrm{TA} V)$.

Proof. Define the projection $f: \mathrm{B}(\mathrm{TA} V ; \mathrm{TA} V ; \mathrm{TA} V) \rightarrow \mathrm{T} V \otimes(\mathbb{k} \oplus s V) \otimes \mathrm{T} V$ on its components $f_{n}: \mathrm{T} V \otimes\left(s \mathrm{~T}^{+} V\right)^{\otimes n} \otimes \mathrm{T} V \rightarrow \mathrm{T} V \otimes(\mathbb{k} \oplus s V) \otimes \mathrm{T} V:$

The map $f_{0}: \mathrm{T} V \otimes \mathrm{T} V \rightarrow \mathrm{T} V \otimes \mathrm{T} V$ is the identity map.

We define $f_{1}: \mathrm{T} V \otimes s \mathrm{~T}^{+} V \otimes \mathrm{T} V \rightarrow \mathrm{T} V \otimes s V \otimes \mathrm{T} V$ by

$$
f_{1}\left(a\left[s v_{1} \cdots v_{n}\right] b\right)=\sum_{i=1}^{n}(-1)^{\left|v_{1} \cdots v_{i-1}\right|} a v_{1} \cdots v_{i-1} \otimes s v_{i} \otimes v_{i+1} \cdots v_{n} b
$$

for $a, b \in \mathrm{T} V, v_{1}, \cdots, v_{n} \in V$ and $n \in \mathbb{N}^{*}$.

For $n \geqslant 2, f_{n}$ is the zero map. An easy calculation shows that $d_{2} f_{1}=f_{0} d_{2}$. Since $f_{1}\left(a\left[s a_{1} a_{2}\right] b\right)=f_{1}\left(a\left[s a_{1}\right] a_{2} b\right)+(-1)^{\left|a_{1}\right|} f_{1}\left(a a_{1}\left[s a_{2}\right] b\right), f_{1} d_{2}=0$. Therefore $f$ commutes with $d_{2}$ and is a morphism of complexes.

Of course, $f \nabla=i d_{\mathrm{T} V \otimes(\mathbb{k} \oplus s V) \otimes \mathrm{T} V}$. The components

$$
\Phi_{n}: \mathrm{T} V \otimes\left(s \mathrm{~T}^{+} V\right)^{\otimes n} \otimes \mathrm{T} V \rightarrow \mathrm{T} V \otimes\left(s \mathrm{~T}^{+} V\right)^{\otimes n+1} \otimes \mathrm{T} V
$$

of the chain homotopy $\Phi$ are defined by:

$$
\begin{aligned}
\Phi_{0}= & 0, \\
\Phi_{n}\left(a\left[s a_{1}|\cdots| s a_{n-1} \mid s v\right] b\right)= & 0, \\
\Phi_{n}\left(a\left[s a_{1}|\cdots| s a_{n-1} \mid s a_{n} v\right] b\right)= & -(-1)^{\varepsilon_{n}} a\left[s a_{1}|\cdots| s a_{n} \mid s v\right] b \\
& +\Phi_{n}\left(a\left[s a_{1}|\cdots| s a_{n}\right] v b\right)
\end{aligned}
$$

for $a, b \in \mathrm{T} V, v \in V$ and $a_{1}, \cdots, a_{n} \in \mathrm{T}^{+} V$. Recall that $\varepsilon_{n}=|a|+\left|s a_{1}\right|+\cdots+\left|s a_{n}\right|$.

By a double induction first on $n$ and then on the wordlength, check that $d \Phi_{n}+$ $\Phi_{n-1} d=\nabla f_{n}-i d, n \in \mathbb{N}$. At the beginning for $n=1$, use the formula $f_{1}\left(a\left[s a_{1} v\right] b\right)=$ $f_{1}\left(a\left[s a_{1}\right] v b\right)+(-1)^{\left|a_{1}\right|} a a_{1} \otimes s v \otimes b$.

Consider now an augmented DGA $(\mathrm{TA} V, \partial)$ such that $\overline{\mathrm{TA} V}=\mathrm{T}^{+} V$. The differential $\partial$ decomposes uniquely as a sum $d_{1}+d_{2}+\cdots+d_{i}+\cdots$ of derivations satisfying $d_{i}(V) \subset \mathrm{T}^{i} V=V^{\otimes i}$. The differential $d_{1}$ is called the linear part of $d$.

To pass from the case $\partial=d_{1}$ to the general case, we'll use the well-known perturbation Lemma. For an abundant and recent bibliography, see [28] or [22].

Theorem 14 (Perturbation Lemma). Let $(X, \partial) \underset{\nabla}{\stackrel{f}{\leftrightarrows}}(Y, d) \circlearrowleft \Phi$ be a strong deformation retract of chain complexes satisfying $f \Phi=0, \Phi \nabla=0$ and $\Phi^{2}=$ 0. Suppose moreover that this strong deformation retract is filtered: there exist on $X$ and on $Y$ increasing filtrations bounded below preserved by $\partial, d, f, \nabla$ and $\Phi$. Consider a filtration-lowering linear map $t: Y \rightarrow Y$ of degree -1 such that $d+t$ is 
a new differential on $Y$ (Such $t$ is called a perturbation). Then

$$
\begin{array}{ll}
\partial_{\infty}=\partial+\sum_{k>0} f(t \Phi)^{k-1} t \nabla, & \nabla_{\infty}=\nabla+\sum_{k>0}(\Phi t)^{k} \nabla, \\
f_{\infty}=f+\sum_{k>0} f(t \Phi)^{k}, & \Phi_{\infty}=\Phi+\sum_{k>0}(\Phi t)^{k} \Phi
\end{array}
$$

are well defined linear maps and $\left(X, \partial_{\infty}\right) \underset{\nabla_{\infty}}{\stackrel{f_{\infty}}{\leftrightarrows}}(Y, d+t) \circlearrowleft \Phi_{\infty}$ is a strong deformation retract.

By applying the Perturbation Lemma to Proposition 13 we rediscover

Theorem 15. [40, Théorème 1.4] Let (TAV,d) be a chain algebra. Suppose that $V$ is a graded module concentrated in degree greater than or equal to one. Define the linear map of degree +1

$$
\begin{aligned}
& S: \mathrm{T} V \otimes \mathrm{T} V \rightarrow \mathrm{T} V \otimes s V \otimes \mathrm{T} V \\
& a \otimes v_{1} \cdots v_{n} \mapsto \sum_{i=1}^{n}(-1)^{\left|a v_{1} \cdots v_{i-1}\right|} a v_{1} \cdots v_{i-1} \otimes s v_{i} \otimes v_{i+1} \cdots v_{n}
\end{aligned}
$$

Consider the chain complex $(\mathrm{TV} \otimes(\mathbb{k} \oplus s V) \otimes \mathrm{T} V, D)$ where

$$
\begin{gathered}
\left.D\right|_{\mathrm{T} V \otimes \mathrm{T} V}=d,\left.\quad D\right|_{\mathrm{T} V \otimes s V \otimes \mathrm{T} V}=\tilde{d}_{1}+d_{2}, \\
\tilde{d}_{1}(a \otimes s v \otimes b)=d a \otimes s v \otimes b-S(a \otimes d v) . b-(-1)^{|a v|} a \otimes s v \otimes d b \\
\text { and } \quad d_{2}(a \otimes s v \otimes b)=(-1)^{|a|}(a v \otimes b-a \otimes v b) \text { for } a, b \in \mathrm{T} V, v \in V .
\end{gathered}
$$

Then $(\mathrm{T} V \otimes(\mathbb{k} \oplus s V) \otimes \mathrm{T} V, D)$ is a strong deformation retract of the (TA $V, \mathrm{TA} V)$ bimodule $\mathrm{B}(\mathrm{TA} V, \mathrm{TA} V$, TA $V)$.

Proof. By Proposition $13\left(\mathrm{~T} V \otimes(\mathbb{k} \oplus s V) \otimes \mathrm{T} V, d_{1}+d_{2}\right)$ is a strong deformation retract of $\mathrm{B}\left(\left(\mathrm{TA} V, d_{1}\right),\left(\mathrm{TA} V, d_{1}\right),\left(\mathrm{TA} V, d_{1}\right)\right)$ where $d_{1}$ denotes the linear part of $d$. The annihilation conditions are satisfied:

$\Phi_{1}(a[s v] b)=0$ and $\Phi_{0}=0$, therefore $\Phi \nabla=0$

The projection $f$ is null on $\mathrm{T} V \otimes\left(s \mathrm{~T}^{+} V\right)^{\otimes \geqslant 2} \otimes \mathrm{T} V$ and $\Phi_{0}=0$. Therefore $f \Phi_{n}=0$ for $n \in \mathbb{N}$.

Since $\Phi_{n+1} \Phi_{n}\left(a\left[s a_{1}|\cdots| s a_{n} v\right] b\right)=\Phi_{n+1} \Phi_{n}\left(a\left[s a_{1}|\cdots| s a_{n}\right] v b\right)$, by induction on wordlength $\Phi_{n+1} \Phi_{n}=0$ for $n \geqslant 1$.

Let $k \in \mathbb{Z}$. An element $a\left[s a_{1}|\cdots| s a_{n}\right] b$ is said to have a filtration degree $-k$ if and only if the sum of the wordlengths of $a, a_{1}, \ldots, a_{n}$ and $b$ is greater than or equal to $k$. The filtrations are bounded below since $V=V_{\geqslant 1}$. The maps $\nabla, f, \Phi, d_{1}$ and $d_{2}$ respect wordlengths. Therefore the strong deformation retract is filtered. Define the perturbation $t$ to be equal to the differential of $\mathrm{B}((\mathrm{TA} V, d),(\mathrm{TA} V, d),(\mathrm{TA} V, d))$ minus the differential of $\mathrm{B}\left(\left(\mathrm{TA} V, d_{1}\right),\left(\mathrm{TA} V, d_{1}\right),\left(\mathrm{TA} V, d_{1}\right)\right)$. Since $d_{\geqslant 2}=d-d_{1}$ increases wordlength by 1 at least, $t$ is filtration-lowering.

So finally we can apply the Perturbation Lemma and $\left(\mathrm{T} V \otimes(\mathbb{k} \oplus s V) \otimes \mathrm{T} V, \partial_{\infty}\right)$ is a strong deformation retract of $\mathrm{B}((\mathrm{TA} V, d),(\mathrm{TA} V, d),(\mathrm{TA} V, d))$. 
The composite $t \Phi_{n}$ maps $\mathrm{T} V \otimes\left(s \mathrm{~T}^{+} V\right)^{\otimes n} \otimes \mathrm{T} V$ into $\mathrm{T} V \otimes\left(s \mathrm{~T}^{+} V\right)^{\otimes n+1} \otimes \mathrm{T} V, \Phi_{0}$ is null. Therefore $f(t \Phi)^{k}=0$ for $k \geqslant 1$. So $f=f_{\infty}$ (The projection is unchanged) and $D:=\partial_{\infty}=\partial+f t \nabla=d_{2}+f d_{1} \nabla$ where $d_{1}$ is the linear part of the differential of $\mathrm{B}((\mathrm{TA} V, d),(\mathrm{TA} V, d),(\mathrm{TA} V, d))$. Set $\tilde{d}_{1}:=f d_{1} \nabla$.

$$
\begin{gathered}
\tilde{d}_{1}(a \otimes s v \otimes b)=d a \otimes s v \otimes b-(-1)^{|a|} f_{1}(a \otimes s d v \otimes b)-(-1)^{|a v|} a \otimes s v \otimes d b . \\
S(a \otimes d v) . b=(-1)^{|a|} f_{1}(a \otimes s d v \otimes b) .
\end{gathered}
$$

Corollary 16. [40, Théorème 1.5] Let $(\mathrm{TA} V, d)$ be a chain algebra such that $V=$ $V_{\geqslant 1}$. Define the linear map of degree +1

$$
\begin{aligned}
\bar{S}: \mathrm{T} V \otimes \mathrm{T} V & \rightarrow s V \otimes \mathrm{T} V \\
v_{1} \ldots v_{n} \otimes a & \mapsto \sum_{i=1}^{n}(-1)^{\left|v_{1} \ldots v_{i-1}\right|\left|v_{i} \ldots v_{n} a\right|} s v_{i} \otimes v_{i+1} \ldots v_{n} a v_{1} \ldots v_{i-1}
\end{aligned}
$$

Consider the complex $((\mathbb{k} \oplus s V) \otimes \mathrm{T} V, \delta)$ where

$$
\begin{gathered}
\left.\delta\right|_{\mathrm{T} V}=d, \\
\delta(s v \otimes a)=(-1)^{|a||v|} 1 \otimes a v-1 \otimes v a+(-1)^{|s v|} s v \otimes d a-\bar{S}(d v \otimes a) .
\end{gathered}
$$

Then $((\mathbb{k} \oplus s V) \otimes \mathrm{T} V, \delta)$ is a strong deformation retract of $\mathrm{C}(\mathrm{TA} V, d)$.

Proof. Tensor by $-\otimes_{\mathrm{TA} V \otimes \mathrm{TA} V}$ op $\mathrm{T} V[\mathbf{4 0}]$.

Suppose that $(\mathrm{TA} V, \partial)$ is a HAH model of a path connected space $X$. Using the inclusion $\nabla_{\infty}$ and the projection $f_{\infty}=f$ of the strong deformation retract given by Corollary 16, it is now easy to transport the diagonal of $\mathrm{C}(\mathrm{TA} V, \partial)$, denoted $\Delta_{\mathrm{C}(\mathrm{TA} V, \partial)}$, to $((\mathbb{k} \oplus s V) \otimes \mathrm{T} V, \delta)$. Define the diagonal of $((\mathbb{k} \oplus s V) \otimes \mathrm{T} V, \delta)$ simply as the composite $(f \otimes f) \circ \Delta_{\mathrm{C}(\mathrm{TA} V, \partial)} \circ \nabla_{\infty}$. Now $((\mathbb{k} \oplus s V) \otimes \mathrm{T} V, \delta)^{\vee}$ is an algebra up to homotopy whose homology is isomorphic to $H H^{*}(\mathrm{TA} V, \partial)$ as graded algebras. This algebra up to homotopy is the smallest that computes in general the cohomology algebra of the free loop space $H^{*}\left(X^{S^{1}}\right)$. But the formula for the diagonal of $((\mathbb{k} \oplus s V) \otimes \mathrm{T} V, \delta)$ is very complicated: it involves in particular the formula for the inclusion $\nabla_{\infty}$ given by the Perturbation Lemma.

We will now limit ourself to two important cases where the HAH structure on $(\mathrm{TA} V, \partial)$ is simple:

- The differential $\partial$ is the sum $d_{1}+d_{2}$ of only its linear part $d_{1}$ and its quadratic part $d_{2}$. The elements of $V$ are primitive: TA $V$ is a primitively generated Hopf algebra. This will be the subject of Section 6 .

- The differential $\partial$ is equal to its linear part $d_{1}$ (hypothesis of Proposition 13). The reduced diagonal $\bar{\Delta}$ of TA $V$ is such that $\bar{\Delta}(V) \subset V \otimes V$. This will be the subject of Section 7. 


\section{The isomorphism between $H H^{*}(\Omega C)$ and $H H_{*}\left(C^{\vee}\right)$}

Let $C$ be a coaugmented DGC. Denote by $\bar{C}$ the kernel of the counit. The cobar construction on $C$, denoted $\Omega C$, is the augmented DGA $\left(\mathrm{TA}\left(s^{-1} \bar{C}\right), d_{1}+d_{2}\right)$ where $d_{1}$ and $d_{2}$ are the unique derivations determined by

$$
\begin{gathered}
d_{1} s^{-1} c=-s^{-1} d c \text { and } \\
d_{2} s^{-1} c=\sum_{i}(-1)^{\left|x_{i}\right|} s^{-1} x_{i} \otimes s^{-1} y_{i}, c \in \bar{C}
\end{gathered}
$$

where the reduced diagonal $\bar{\Delta} c=\sum_{i} x_{i} \otimes y_{i}$. We follow the sign convention of [16]. When restricted to conilpotent coaugmented DGC's, the cobar construction $\Omega$ is a left adjoint functor to the bar construction B [16, Proposition 2.11]. Denote by $\sigma_{C}: C \stackrel{\widetilde{ }}{\rightarrow} \mathrm{B} \Omega C$ the adjunction map.

Theorem 17. (Compare with [26, Theorem A], [21, Theorem II] or [38, Theorem 1.1]) Consider a coaugmented $D G C C \mathbb{k}$-free of finite type such that $C=\mathbb{k} \oplus C_{\geqslant 2}$. Then there is a chain complex of the form $(C \otimes \Omega C, \delta)$ such that:

i) The composite

$$
(C \otimes \Omega C, \delta) \stackrel{\tau_{C, \Omega C}}{\cong} \Omega C \otimes C \stackrel{\Omega C \otimes \sigma_{C}}{\simeq} \Omega C \otimes \mathrm{B}(\Omega C)=\mathrm{C}(\Omega C)
$$

is a quasi-isomorphism of chain complexes.

ii) The dual of this complex $(C \otimes \Omega C, \delta)$ is isomorphic to $\mathrm{C}\left(C^{\vee}\right)$.

In particular, there is a natural isomorphism of graded modules

$$
H H^{*}(\Omega C) \cong H H_{*}\left(C^{\vee}\right) \text {. }
$$

We give again the proof of Jones and McCleary since we want to check carefully the signs. We have explicited the isomorphism in order to transport later the algebra structure. Remark that already at the level of complexes, there is a quasiisomorphism from $\mathrm{C}(\Omega C)^{\vee}$ to $\mathrm{C}\left(C^{\vee}\right)$.

Before beginning the proof, we precise the signs convention used in this paper: We have chosen to make constant use of the Koszul sign convention. For example, let $\varphi: M \rightarrow N$ be a linear map. If $f \in \operatorname{Hom}(N, \mathbb{k})$ then

$$
\varphi^{\vee}(f)=(-1)^{|f||\varphi|} f \circ \varphi .
$$

In particular, if $(M, d)$ is a complex, the dual complex is $\left(M^{\vee}, d^{\vee}\right)$.

Proof. By Formula 1, the adjunction map $\sigma_{C}: C \stackrel{\simeq}{\rightarrow} \mathrm{B} \Omega C$ is given by

$$
\sigma_{C}(c)=\sum_{i=0}^{+\infty} \sum\left[s s^{-1} c_{1}|\cdots| s s^{-1} c_{i+1}\right], c \in \bar{C}
$$

where the iterated reduced diagonal $\bar{\Delta}^{i} c=\sum c_{1} \otimes \cdots \otimes c_{i+1}$. We consider the inclusion map

$$
\nabla_{\infty}=\nabla+\sum_{k>0}(\Phi t)^{k} \nabla
$$


of the strong deformation retract given by Theorem 15 when the chain algebra $(\mathrm{TA} V, d)$ is the cobar construction $\Omega C$. Since the perturbation $t$ is here the tensorization of the $d_{2}$ of the cobar construction, using the formulas for $\Phi$ given in the proof of Proposition 13, an induction on $k$ shows that $\nabla_{\infty}$ is $\Omega C \otimes \sigma_{C} \otimes \Omega C$, the tensor product of the identity maps and the adjunction map. Tensorization by $-\otimes_{\Omega C} \otimes \Omega C^{o p} \Omega C$ gives i).

We now check ii). By applying Corollary 16 when the chain algebra (TA $V, d)$ is the cobar construction $\Omega C$, we obtain a strong deformation retract of the cyclic bar construction $\mathrm{C}(\Omega C)$ of the form $(C \otimes \Omega C, \delta)$. The differential $\delta$ is given by

$$
\begin{aligned}
\delta a= & d_{\Omega} a, a \in \Omega C, \\
\delta(c \otimes a)= & d c \otimes a+(-1)^{|c|} c \otimes d a \\
& -1 \otimes\left(s^{-1} c\right) a-(-1)^{\left|x_{i}\right|} x_{i} \otimes\left(s^{-1} y_{i}\right) a \\
& +(-1)^{|a|\left|s^{-1} c\right|} 1 \otimes a s^{-1} c+(-1)^{\left(|a|+\left|y_{i}\right|\right)\left|s^{-1} x_{i}\right|} y_{i} \otimes a s^{-1} x_{i} .
\end{aligned}
$$

Therefore $(C \otimes \Omega C, \delta)$ is the complex $\left(C \otimes \mathrm{T}^{-1} \bar{C}, d_{1}+d_{2}\right)$ where $d_{1}$ is just obtained by tensorization and $d_{2}: C \otimes\left(s^{-1} \bar{C}\right)^{\otimes n-1} \rightarrow C \otimes\left(s^{-1} \bar{C}\right)^{\otimes n}$ is the sum of $n+1$ terms $\delta_{0}, \delta_{1}, \ldots, \delta_{n}$ given by

$$
\begin{gathered}
\delta_{0}=-\left[\left(C \otimes s^{-1}\right) \circ \Delta\right] \otimes\left(s^{-1} \bar{C}\right)^{\otimes n-1}, \\
\delta_{i}=C \otimes\left(s^{-1} \bar{C}\right)^{\otimes i-1} \otimes\left[\left(s^{-1} \otimes s^{-1}\right) \circ \Delta \circ s\right] \otimes\left(s^{-1} \bar{C}\right)^{\otimes n-1-i}, 1 \leqslant i \leqslant n-1 \\
\text { and } \delta_{n}=\left[C \otimes\left(s^{-1} \bar{C}\right)^{\otimes n-1} \otimes s^{-1}\right] \circ \tau_{C, C \otimes\left(s^{-1} \bar{C}\right)^{\otimes n-1}} \circ\left[\Delta \otimes\left(s^{-1} \bar{C}\right)^{\otimes n-1}\right] .
\end{gathered}
$$

Let $A$ denote the augmented DGA $C^{\vee}$. The differential $d_{2}: A \otimes(s \bar{A})^{\otimes n} \rightarrow A \otimes$ $(s \bar{A})^{\otimes n-1}$ is also the sum of $n+1$ terms $d_{0}, d_{1}, \ldots, d_{n}$ (compare to Example 3 ) given by

$$
\begin{gathered}
d_{0}=\left[\mu \circ\left(A \otimes s^{-1}\right)\right] \otimes(s \bar{A})^{\otimes n-1}, \\
d_{i}=A \otimes(s \bar{A})^{\otimes i-1} \otimes\left[s \circ \mu \circ\left(s^{-1} \otimes s^{-1}\right)\right] \otimes(s \bar{A})^{n-i-1}, 1 \leqslant i \leqslant n-1 \\
\text { and } d_{n}=-\left[\mu \otimes(s \bar{A})^{\otimes n-1}\right] \circ \tau_{A \otimes(s \bar{A})^{\otimes n-1}, A} \circ\left[A \otimes(s \bar{A})^{\otimes n-1} \otimes s^{-1}\right] .
\end{gathered}
$$

The isomorphism $\Theta: s\left(\bar{C}^{\vee}\right) \stackrel{\cong}{\rightrightarrows}\left(s^{-1} \bar{C}\right)^{\vee}$ is such that $\left(s^{-1}\right)^{\vee} \circ \Theta=s^{-1}[\mathbf{2 0}$, p. 276]. For any two complexes $V$ and $W$, the map $\Phi: V^{\vee} \otimes W^{\vee} \rightarrow(V \otimes W)^{\vee}$ given by $\Phi(f \otimes g)=\mu_{\mathbb{k}} \circ(f \otimes g)$ is a morphism of complexes and is associative, commutative, natural with respect to linear maps of any degree. Therefore the composite

$$
A \otimes(s \bar{A})^{\otimes n} \stackrel{A \otimes \Theta^{\otimes n}}{\longrightarrow} C^{\vee} \otimes\left[\left(s^{-1} \bar{C}\right)^{\vee}\right]^{\otimes n} \stackrel{\Phi}{\rightarrow}\left[C \otimes\left(s^{-1} \bar{C}\right)^{\otimes n}\right]^{\vee}
$$

commutes with $d_{i}$ and $\delta_{i}^{\vee}$ for $0 \leqslant i \leqslant n$. So finally

$$
\Phi \circ[A \otimes \mathrm{T}(\Theta)]: \mathrm{C}(A) \stackrel{\cong}{\rightrightarrows}(C \otimes \Omega C, \delta)^{\vee}
$$

is an isomorphism of complexes.

Let $V$ be a graded module. The tensor algebra TA $V$ can be made into a cocommutative Hopf algebra by requiring the elements of $V$ to be primitive [39, $0.5(10)]$. 
We will call the resulting diagonal the shuffle diagonal. Dually the tensor coalgebra TCV equipped with the shuffle product is a commutative Hopf algebra. The shuffle product is defined by

$$
\left[v_{1}|\ldots| v_{p}\right] \cdot\left[v_{p+1}|\ldots| v_{p+q}\right]=\sum_{\sigma} \sigma \cdot\left[v_{1}|\ldots| v_{p+q}\right]
$$

where the sum is taken over the $(p, q)$-shuffles $\sigma$ and a permutation $\sigma$ acts on $\left[v_{1}|\ldots| v_{p+q}\right]$ by permuting the factors with appropriate signs ([20, Appendix] or $[39,0.5(8)])$. Suppose that $V$ is $\mathbb{k}$-free of finite type and $V=V_{\geqslant 1}$. Then the map $\Phi: \operatorname{TC}\left(V^{\vee}\right) \cong(\mathrm{TA} V)^{\vee}$ is an isomorphism of Hopf algebras.

Let $C$ be a cocommutative coaugmented DGC. Then the cobar $\Omega C=$ $\left(\mathrm{TA}\left(s^{-1} \bar{C}\right), d_{1}+d_{2}\right)$ equipped with the shuffle diagonal is a DGH $[\mathbf{3 9}, 0.6(2)]$. Dually, let $A$ be an augmented CDGA. Consider the multiplication on $A \otimes \mathrm{TC}(s \bar{A}) \otimes A$ obtained by tensoring the multiplication of $A$ and the shuffle product of $\operatorname{TC}(s \bar{A})$. Then the bar resolution of $A, \mathrm{~B}(A ; A ; A)=\left(A \otimes \mathrm{TC}(s \bar{A}) \otimes A, d_{1}+d_{2}\right)$ is a CDGA. The cyclic bar construction $\mathrm{C}(A)=\left(A \otimes \mathrm{TC}(s \bar{A}), d_{1}+d_{2}\right)$ is also a CDGA. Therefore the Hochschild homology of a CDGA $A, H H_{*}(A)$, has a natural structure of commutative graded algebra $[\mathbf{2 9}, 4.2 .7]$. The reduced bar construction of $A$, $\mathrm{B}(A)=\left(\mathrm{TC}(s \bar{A}), d_{1}+d_{2}\right)$ is a commutative DGH $[\mathbf{3 9}, 0.6(1)]$.

Theorem 18. Under the hypothesis of Theorem 17, if $C$ is cocommutative then the isomorphism $H H^{*}(\Omega C) \cong H H_{*}\left(C^{\vee}\right)$ is an isomorphism of commutative graded algebras.

This theorem has by now been extended by Idrissi $[\mathbf{2 4}]$ to the more general case where $C$ is a "strongly homotopy commutative" coalgebra.

Property 19. Let $K$ be a graded Hopf algebra. Consider Ker $\bar{\Delta}$ the primitive elements of $K$ and the graded coalgebra $K \otimes \mathrm{TC}(s \operatorname{Ker} \bar{\Delta}) \otimes K$ obtained by tensorization. Then the canonical map $K \otimes \mathrm{TC}(s \operatorname{Ker} \bar{\Delta}) \otimes K \rightarrow \mathrm{B}(K ; K ; K)$ is a morphism of graded coalgebras.

Proof of Theorem 18. In the proof of Theorem 17, we have seen that

$$
\Omega C \otimes \sigma_{C} \otimes \Omega C:(\Omega C \otimes C \otimes \Omega C, \delta) \stackrel{\simeq}{\hookrightarrow} \mathrm{B}(\Omega C ; \Omega C ; \Omega C)
$$

is a strong deformation retract. The adjunction map $\sigma_{C}$ is the composite of two morphisms of graded coalgebras:

$$
C \stackrel{\sigma_{1}}{\rightarrow} \mathrm{TC}\left(s s^{-1} \bar{C}\right) \stackrel{\sigma_{2}}{\hookrightarrow} \mathrm{TC}\left[s \mathrm{~T}^{+}\left(s^{-1} \bar{C}\right)\right]=\mathrm{B} \Omega C .
$$

Consider the coalgebra structures obtained by tensorization on $\Omega C \otimes C \otimes \Omega C$ and $\Omega C \otimes \mathrm{TC}\left(s s^{-1} \bar{C}\right) \otimes \Omega C$. Obviously

$$
\Omega C \otimes \sigma_{1} \otimes \Omega C: \Omega C \otimes C \otimes \Omega C \rightarrow \Omega C \otimes \mathrm{TC}\left(s s^{-1} \bar{C}\right) \otimes \Omega C
$$

is a morphism of graded coalgebras. Since $s^{-1} \bar{C} \subset \operatorname{Ker} \bar{\Delta}_{\Omega C}$, by Property 19 ,

$$
\Omega C \otimes \sigma_{2} \otimes \Omega C: \Omega C \otimes \mathrm{TC}\left(s s^{-1} \bar{C}\right) \otimes \Omega C \hookrightarrow \mathrm{B}(\Omega C ; \Omega C ; \Omega C)
$$

is an inclusion of graded coalgebras. Finally, we have proved that $\Omega C \otimes \sigma_{C} \otimes \Omega C$ is both a morphism of complexes and of graded coalgebras. Since $\left(\Omega C \otimes \sigma_{C} \otimes \Omega C\right)^{\otimes 2}$ 
is a monomorphism, the coalgebra $\Omega C \otimes C \otimes \Omega C$ obtained by tensorization is a sub DGC of $\mathrm{B}(\Omega C ; \Omega C ; \Omega C)$. After tensoring by $-\otimes_{\Omega C} \otimes \Omega C^{o p} \Omega C$ and dualizing, we obtain the natural DGA quasi-isomorphisms

$$
\mathrm{C}\left(C^{\vee}\right) \stackrel{\cong}{\rightrightarrows}(C \otimes \Omega C, \delta)^{\vee} \stackrel{\cong}{ } \mathrm{C}(\Omega C)^{\vee} .
$$

\section{The free loop space on a suspension}

In this section, we show how to compute the cohomology algebra of the free loop space on any suspension, $H^{*}\left((\Sigma Y)^{S^{1}}\right)$ (Theorem 27). A more precise result is given in Theorem 25: we construct a finite type cochain algebra weakly equivalent to $S^{*}\left((\Sigma Y)^{S^{1}}\right)$.

First we introduce some terminology. Let $C$ be a coaugmented DGC. The composite $C \stackrel{\Delta_{C}}{\longrightarrow} C \otimes C \hookrightarrow \mathrm{TA} \bar{C} \otimes \mathrm{TA} \bar{C}$ extends to an unique morphism of augmented DGA's

$$
\Delta_{\mathrm{TA}}: \mathrm{TA} \bar{C} \rightarrow \mathrm{TA} \bar{C} \otimes \mathrm{TA} \bar{C} .
$$

This DGH structure on the tensor algebra TA $\bar{C}$ is called the Hopf algebra structure obtained by tensorization of the coalgebra $C$. Dually, let $A$ be an augmented DGA. The composite $\mathrm{TC} \bar{A} \otimes \mathrm{TC} \bar{A} \rightarrow A \otimes A \stackrel{\mu_{A}}{\rightarrow} A$ lifts to an unique morphism of coaugmented DGC's

$$
\mu_{\mathrm{TC} \bar{A}}: \mathrm{TC} \bar{A} \otimes \mathrm{TC} \bar{A} \rightarrow \mathrm{TC} \bar{A} .
$$

This DGH structure on the tensor coalgebra TC $\bar{A}$ is called the Hopf algebra structure obtained by tensorization of the algebra $A$. Using formula 1 , we see that the product $\mu_{\mathrm{TC} \bar{A}}$ of two elements $\left[a_{1}|\cdots| a_{p}\right]$ and $\left[b_{1}|\cdots| b_{q}\right]$ admits the following description:

A sequence $\sigma=\left((0,0)=\left(x_{0}, y_{0}\right),\left(x_{1}, y_{1}\right), \ldots,\left(x_{n}, y_{n}\right)=(p, q)\right)$ defined by

$$
\left(x_{i}, y_{i}\right)= \begin{cases}\left(x_{i-1}+1, y_{i-1}\right) & \text { or } \\ \left(x_{i-1}, y_{i-1}+1\right) & \text { or } \\ \left(x_{i-1}+1, y_{i-1}+1\right), & \end{cases}
$$

is called a step by step path of length $n$ from $(0,0)$ to $(p, q)$. To any step by step path $\sigma$ of length $n$, we associate $c_{\sigma}=\left[c_{1}|\cdots| c_{n}\right] \in \bar{A}^{\otimes n}$ by the rule

$$
c_{i}= \begin{cases}a_{x_{i}} & \text { if }\left(x_{i}, y_{i}\right)=\left(x_{i-1}+1, y_{i-1}\right), i^{t h} \text { step is toward right, } \\ b_{y_{i}} & \text { if }\left(x_{i}, y_{i}\right)=\left(x_{i-1}, y_{i-1}+1\right), i^{\text {th }} \text { step is toward up, } \\ \mu_{A}\left(a_{x_{i}} \otimes b_{y_{i}}\right) & \text { if }\left(x_{i}, y_{i}\right)=\left(x_{i-1}+1, y_{i-1}+1\right), i^{\text {th }} \text { step in diagonal. }\end{cases}
$$

Then a straightforward computation establishes

$$
\mu_{\mathrm{TC} \bar{A}}\left(\left[a_{1}|\cdots| a_{p}\right] \otimes\left[b_{1}|\cdots| b_{q}\right]\right)=\sum_{\sigma} \pm c_{\sigma}
$$

where the sum is taken over all the step by step paths $\sigma$ from $(0,0)$ to $(p, q)$ and where \pm is the sign obtained with Koszul rule by mixing the $a_{1}, \cdots, a_{p}$ and the 
$b_{1}, \cdots, b_{q}$. In particular, when the product of $A$ is trivial, the product $\mu_{\mathrm{TC}} \bar{A}$ is the shuffle product considered on page 210 .

Suppose that the DGC $C$ is $\mathbb{k}$-free of finite type and such that $C=\mathbb{k} \oplus C_{\geqslant 1}$. Then the map

$$
\Phi: \operatorname{TC}\left(\overline{C^{\vee}}\right) \cong(\mathrm{TA} \bar{C})^{\vee}
$$

is a DGH isomorphism.

The starting observations of this section are the following consequences of the Bott-Samelson Theorem (see [23, A2.Theorems 1.4 and 1.5] and [34, 7.1] for details).

Lemma 21. Let $Y$ be a path connected space. Consider the Hopf algebra structure on $\mathrm{TA} \overline{S_{*}(Y)}$ obtained by tensorization of the coalgebra $S_{*}(Y)$. Then there is a natural DGH quasi-isomorphism

$$
\mathrm{TA} \overline{S_{*}(Y)} \stackrel{\cong}{\rightarrow} S_{*}(\Omega \Sigma Y) .
$$

Lemma 22. Let $Y$ be a path connected space such that $H_{*}(Y)$ is $\mathbb{k}$-free of finite type. Consider the Hopf algebra structure on $\mathrm{TAH}_{+}(Y)$ obtained by tensorization on the coalgebra $H_{*}(Y)$. Then there is a HAH quasi-isomorphism

$$
\Theta_{Y}: \mathrm{TA}_{+}(Y) \stackrel{\simeq}{\rightrightarrows} S_{*}(\Omega \Sigma Y) .
$$

In particular, the Hopf algebra on $\mathrm{TCH}^{+}(Y)$ obtained by tensorization of the algebra $H^{*}(Y)$ is isomorphic to $H^{*}(\Omega \Sigma Y)$.

We can choose $\Theta_{Y}$ to be natural in homology. By the lifting lemma $[\mathbf{1 7}, 3.6]$, such $\Theta_{Y}$ is natural up to homotopy of algebras.

Lemma 23. Let $C$ be a chain coalgebra $\mathbb{k}$-free of finite type such that $C=\mathbb{k} \oplus C_{\geqslant 1}$. Consider the Hopf algebra structure on $\mathrm{TA} \bar{C}$ obtained by tensorization of the coalgebra $C$. Denote by $A$ the cochain algebra dual of $C$. Then there is a natural structure of DGA on the cyclic bar construction $\mathrm{C}\left(\mathbb{k} \oplus s^{-1} \bar{A}\right)$ on the trivial cochain algebra $\left(\mathbb{k} \oplus s^{-1} \bar{A}\right)$ such that the quasi-isomorphism of complexes given by Theorem 17

$$
\mathrm{C}(\mathrm{TA} \bar{C})^{\vee} \stackrel{\simeq}{\rightarrow} \mathrm{C}\left(\mathbb{k} \oplus s^{-1} \bar{A}\right)
$$

becomes a DGA quasi-isomorphism. The product on $\mathrm{C}\left(\mathbb{k} \oplus s^{-1} \bar{A}\right)$ satisfies the following definition:

Definition 24. Let $A$ be an augmented DGA. Consider the Hopf algebra structure on TC $\bar{A}$ obtained by tensorization of the algebra $A$. Consider the complex $\left(\mathbb{k} \oplus s^{-1} \bar{A}\right)$ 
equipped with trivial product. The formulas

$$
\begin{aligned}
(1 \otimes m) \cdot\left(1 \otimes m^{\prime}\right)= & 1 \otimes \mu_{\mathrm{TC} \bar{A}}\left(m \otimes m^{\prime}\right), \\
\left(s^{-1} a \otimes m\right) .\left(1 \otimes a_{1} \ldots a_{n}\right)= & s^{-1} a \otimes \mu_{\mathrm{TC} \bar{A}}\left(m \otimes a_{1} \ldots a_{n}\right) \\
& \pm s^{-1} \mu_{A}\left(a \otimes a_{n}\right) \otimes \mu_{\mathrm{TC} \bar{A}}\left(m \otimes a_{1} \ldots a_{n-1}\right), \\
\left(1 \otimes a_{1} \ldots a_{n}\right) .\left(s^{-1} a \otimes m\right)= & \pm s^{-1} a \otimes \mu_{\mathrm{TC} \bar{A}}\left(a_{1} \ldots a_{n} \otimes m\right) \\
& \pm s^{-1} \mu_{A}\left(a_{1} \otimes a\right) \otimes \mu_{\mathrm{TC} \bar{A}}\left(a_{2} \ldots a_{n} \otimes m\right)
\end{aligned}
$$

and

$$
\left(s^{-1} a \otimes m\right) \cdot\left(s^{-1} a^{\prime} \otimes m^{\prime}\right)=0 .
$$

for $a, a^{\prime} \in \bar{A}, m, m^{\prime}$ and $a_{1} \ldots a_{n} \in \mathrm{T} \bar{A}$ and where \pm are the signs obtained exactly by the Koszul sign convention, define a product on $\mathrm{C}\left(\mathbb{k} \oplus s^{-1} \bar{A}\right)=\left(\mathbb{k} \oplus s^{-1} \bar{A}\right) \otimes \mathrm{T} \bar{A}$, the cyclic bar construction on $\left(\mathbb{k} \oplus s^{-1} \bar{A}\right)$. We call this product on $\mathrm{C}\left(\mathbb{k} \oplus s^{-1} \bar{A}\right)$ and the resulting product in $H H_{*}\left(\mathbb{k} \oplus s^{-1} \bar{A}\right)$, the products perturbed by the algebra $A$. If the product on $A$ is trivial, these products reduce to the shuffle products.

We can now state the main results of this section.

Theorem 25. Let $Y$ be a path connected space. If $S_{*}(Y)$ is weakly equivalent as $\mathbb{k}$-free chain coalgebra to a chain coalgebra $C \mathbb{k}$-free of finite type such that $C=$ $\mathbb{k} \oplus C_{\geqslant 1}$. Then the singular cochains on the free loop spaces on the suspension of $Y$, $S^{*}\left((\Sigma Y)^{S^{1}}\right)$ is weakly equivalent as cochain algebras to the cyclic bar construction $\mathrm{C}\left(\mathbb{k} \oplus s^{-1} \overline{C^{\vee}}\right)$ equipped with the product perturbed by the algebra $C^{\vee}$.

Remark 26. If $\mathrm{Y}$ is a simply connected $\mathrm{CW}$-complex of finite type then $Y$ satisfies the hypothesis of Theorem 25. Indeed, $S_{*}(Y)$ is weakly equivalent as $\mathbb{k}$-free chain coalgebras to the bar construction $\mathrm{B} S_{*}(\Omega Y)$ and so to the bar construction of the Adams-Hilton model of $Y, \mathrm{~B} \mathcal{A}(Y)$.

Theorem 27. Let $Y$ be a path connected space such that $H_{*}(Y)$ is $\mathbb{k}$-free of finite type. Consider the Hochschild homology on $H^{*}(\Sigma Y), H H_{*}\left(\mathbb{k} \oplus s^{-1} H^{+}(Y)\right)$, equipped with the product perturbed by the algebra $H^{*}(Y)$ (Definition 24). Then there is natural isomorphism of graded algebras $H^{*}\left((\Sigma Y)^{S^{1}}\right) \cong H H_{*}\left(H^{*}(\Sigma Y)\right)$.

It is worth noting the following particular case of Theorem 27.

Corollary 28. Let $Y$ be a path connected space such that $H_{*}(Y)$ is $\mathbb{k}$-free of finite type. If the cup product on $H^{*}(Y)$ is trivial, then $H^{*}\left((\Sigma Y)^{S^{1}}\right)$ is naturally isomorphic as graded algebras to $H_{*}\left(H^{*}(\Sigma Y)\right)$ equipped with the shuffle product.

This Corollary of Theorem 27 can be proved more easily by applying just Lemma 22, Theorem 11 and Theorem 18.

We show now that the algebra $H^{*}\left((\Sigma Y)^{S^{1}}\right)$ has a particular form.

Let $V$ be a graded module. The circular permutation to the right $\tau$ acts on $\mathrm{T}^{n} V$ by

$$
\tau \cdot\left[v_{1}|\ldots| v_{n}\right]=(-1)^{\left|v_{n}\right|\left|v_{1} \ldots v_{n-1}\right|}\left[v_{n}\left|v_{1}\right| \ldots \mid v_{n-1}\right]
$$


Define the module of invariants under $\tau$ by

$$
\mathrm{T}^{n} V^{\tau}=\left\{x \in \mathrm{T}^{n} V, \tau . x=x\right\} \quad \text { and } \quad \mathrm{T}^{\tau}=\bigoplus_{n=0}^{\infty} \mathrm{T}^{n} V^{\tau} .
$$

Define the module of coinvariants under $\tau$ by

$$
\mathrm{T}^{n} V_{\tau}=\frac{\mathrm{T}^{n} V}{\left\{x-\tau \cdot x, x \in \mathrm{T}^{n} V\right\}} \quad \text { and } \quad \mathrm{T} V_{\tau}=\bigoplus_{n=0}^{\infty} \mathrm{T}^{n} V_{\tau} .
$$

Definition 29. [36, 2.2.11] Let $R$ be a graded algebra. Let $M$ be a $(R, R)$-bimodule. The graded module $R \oplus M$, product of $R$ and of $M$ equipped with the multiplication

$$
\left(r_{1}, m_{1}\right)\left(r_{2}, m_{2}\right)=\left(r_{1} r_{2}, r_{1} \cdot m_{2}+m_{1} \cdot r_{2}\right)
$$

is a graded algebra called the trivial extension of $R$ by $M$.

Remark that a graded algebra of the form $R \oplus M$ where $R$ and $M$ are two graded modules is a trivial extension of $R$ by $M$ if and only if $R$ is a subalgebra of $R \oplus M$ and the product of elements of $M$ is always null.

Property 30. Consider a DGA of the form $(R \oplus M, d)$ where the underlying graded algebra is a trivial extension of a graded algebra $R$ by a $(R, R)$-bimodule $M$. If the differential $d$ satisfies

$$
d(R) \subset M \quad \text { and }\left.\quad d\right|_{M}=0 .
$$

Then

i) $\operatorname{Ker}\left(\left.d\right|_{R}: R \rightarrow M\right)$ is a graded subalgebra of $R$.

ii) The graded algebra $H(R \oplus M, d)$ is a trivial extension of $\operatorname{ker}\left(\left.d\right|_{R}: R \rightarrow M\right)$ by $\operatorname{coker}\left(\left.d\right|_{R}: R \rightarrow M\right)$.

Corollary 31. Let $Y$ be a path connected space such that $H_{*}(Y)$ is $\mathbb{k}$-free of finite type.

i) The invariants of the Hopf algebra $\mathrm{TC}^{+}(Y) \cong H^{*}(\Omega \Sigma Y)$ form a graded subalgebra, denoted $\mathrm{TCH} H^{+}(Y)^{\tau}$.

ii) The algebra $H^{*}\left((\Sigma Y)^{S^{1}}\right)$ is isomorphic to a trivial extension of $\operatorname{TCH} H^{+}(Y)^{\tau}$ by $s^{-1}\left[\mathrm{~T}^{+} H^{+}(Y)_{\tau}\right]$, that is the graded module of coinvariants of positive length up to a desuspension of degree.

When $\mathbb{k}=\mathbb{Q}$ and $Y$ is a wedge of spheres, this Corollary has been proved by Parhizgar [36, Theorems 2.2.4 and 3.2.2].

Proof. The cyclic bar construction on $\left(\mathbb{k} \oplus s^{-1} H^{+}(Y)\right), \mathrm{C}\left(H^{*}(\Sigma Y)\right)$, equipped with the product perturbed by the algebra $H^{*}(Y)$, is a cochain algebra (Lemma 23). The underlying graded algebra of $\mathrm{C}\left(H^{*}(\Sigma Y)\right)$ is a trivial extension of $R=\mathrm{TC} H^{+}(Y)$ by

$$
M=s^{-1} H^{+}(Y) \otimes \mathrm{T} H^{+}(Y)=s^{-1}\left[\mathrm{~T}^{+} H^{+}(Y)\right] .
$$


The differential $d$ of $\mathrm{C}\left(H^{*}(\Sigma Y)\right)$ is given by

$$
\begin{aligned}
d\left(s^{-1} y\left[y_{1}|\ldots| y_{n}\right]\right) & =0 \text { and } \\
d\left(\left[y_{1}|\ldots| y_{n}\right]\right) & =s^{-1} y_{1}\left[y_{2}|\ldots| y_{n}\right]-s^{-1} \tau \cdot\left[y_{1}|\ldots| y_{n}\right]
\end{aligned}
$$

for $y, y_{1}, \ldots, y_{n} \in H^{+}(Y)$. Therefore, by Property 30, the Hochschild homology on $H^{*}(\Sigma Y), H H_{*}\left(\mathbb{k} \oplus s^{-1} H^{+}(Y)\right)$, equipped with the product perturbed by the algebra $H^{*}(Y)$ is a trivial extension of $\mathrm{TCH}^{+}(Y)^{\tau}$ by $s^{-1}\left[\mathrm{~T}^{+} H^{+}(Y)_{\tau}\right]$. By Theorem 27, this trivial extension is isomorphic to the algebra $H^{*}\left((\Sigma Y)^{S^{1}}\right)$.

Property 32. Let $K$ be a graded Hopf algebra. The diagonal of the coalgebra $\mathrm{B}(K ; K ; K)$ restricted to $K \otimes(\mathbb{k} \oplus s \bar{K}) \otimes K$ is the $(K, K)$-linear map given by

$$
\Delta[s x]=[s x] \otimes[]+[s y] \otimes z[]+(-1)^{|y|}[] y \otimes[s z]+[] \otimes[s x], x \in \bar{K}
$$

where the reduced diagonal $\bar{\Delta} x=\sum y \otimes z$.

Proof of Lemma 23. The tensor algebra TA $\bar{C}$ is equal as DGA to the cobar on the DGC $\mathbb{k} \oplus s \bar{C}$ with trivial coproduct. Therefore by Theorem 17 , we get immediately a natural quasi-isomorphism of cochain complexes

$$
\mathrm{C}\left(\mathbb{k} \oplus s^{-1} \bar{A}\right) \stackrel{\simeq}{=} \mathrm{C}(\mathrm{TA} \bar{C})^{\vee} .
$$

But we have to remember how this morphism decomposes in order to transport the multiplication from $\mathrm{C}(\mathrm{TA} \bar{C})^{\vee}$ to $\mathrm{C}\left(\mathbb{k} \oplus s^{-1} \bar{A}\right)$.

Since the differential on TA $\bar{C}$ is only linear, by Proposition 13, the canonical inclusion

$$
\mathrm{T} \bar{C} \otimes(\mathbb{k} \oplus s \bar{C}) \otimes \mathrm{T} \bar{C} \stackrel{\sim}{\hookrightarrow} \mathrm{B}(\mathrm{TA} \bar{C} ; \mathrm{TA} \bar{C} ; \mathrm{TA} \bar{C})
$$

is a quasi-isomorphism of complexes. Since the reduced diagonal of TA $\bar{C}, \bar{\Delta}_{\mathrm{TA}} \bar{C}$ embeds $\bar{C}$ into $\bar{C} \otimes \bar{C}$, by Property $32, \mathrm{~T} \bar{C} \otimes(\mathbb{k} \oplus s \bar{C}) \otimes \mathrm{T} \bar{C}$ is a sub DGC of $\mathrm{B}(\mathrm{TA} \bar{C} ; \mathrm{TA} \bar{C} ; \mathrm{TA} \bar{C})$. By tensoring by $-\otimes_{\mathrm{TA}} \bar{C} \otimes \mathrm{TA} \bar{C} \mathrm{TA} \bar{C}$, we obtain the DGC (k $\oplus$ $s \bar{C}) \otimes \mathrm{T} \bar{C}$ with differential given by Corollary 16 and diagonal given by

$$
\begin{aligned}
\Delta(s x \otimes c)= & s x \otimes c^{\prime} \otimes 1 \otimes c^{\prime \prime}+(-1)^{|c||z|} s y \otimes c^{\prime} \otimes 1 \otimes c^{\prime \prime} z \\
& +(-1)^{|y|+\left|c^{\prime}\right||s z|} 1 \otimes y c^{\prime} \otimes s z \otimes c^{\prime \prime}+(-1)^{\left|c^{\prime}\right||s x|} 1 \otimes c^{\prime} \otimes s x \otimes c^{\prime \prime}
\end{aligned}
$$

for $x \in \bar{C}, c \in \mathrm{T} \bar{C}$ and where the reduced diagonal $\bar{\Delta} x=\sum y \otimes z$ and the unreduced diagonal $\Delta c=\sum c^{\prime} \otimes c^{\prime \prime}$. The canonical inclusion

$$
(\mathbb{k} \oplus s \bar{C}) \otimes \mathrm{T} \bar{C} \stackrel{\sim}{\hookrightarrow} \mathrm{C}(\mathrm{TA} \bar{C})
$$

is a DGC quasi-isomorphism.

In order to dualize (for details, review the proof of Theorem 17), we see that the diagonal on $(\mathbb{k} \oplus s \bar{C}) \otimes \mathrm{T} \bar{C}$ is the sum of three terms,

$$
\Delta_{\mathrm{TA} \bar{C}}: \mathrm{T} \bar{C} \rightarrow \mathrm{T} \bar{C} \otimes \mathrm{T} \bar{C},
$$

$$
\Delta_{1}: s \bar{C} \otimes \mathrm{T} \bar{C} \rightarrow s \bar{C} \otimes \mathrm{T} \bar{C} \otimes \mathrm{T} \bar{C}
$$

and

$$
\Delta_{2}: s \bar{C} \otimes \mathrm{T} \bar{C} \rightarrow \mathrm{T} \bar{C} \otimes s \bar{C} \otimes \mathrm{T} \bar{C} .
$$


The first term $\Delta_{\mathrm{TA}} \bar{C}$ is just the diagonal of TA $\bar{C}$. The second term $\Delta_{1}$ is the composite

$\left(s \otimes \mathrm{T} \bar{C} \otimes \mu_{\mathrm{TA} \bar{C}}\right) \circ(C \otimes \mathrm{T} \bar{C} \otimes \mathrm{T} \bar{C} \otimes i) \circ\left(C \otimes \tau_{C, \mathrm{~T}} \bar{C} \otimes \mathrm{T} \bar{C}\right) \circ\left(\Delta_{C} \otimes \Delta_{\mathrm{TA}} \bar{C}\right) \circ\left(s^{-1} \otimes \mathrm{T} \bar{C}\right)$

where $i$ denotes the inclusion $C \hookrightarrow \mathrm{T} \bar{C}$. The third term $\Delta_{2}$ is the composite

$$
\left(\mu_{\mathrm{TA} \bar{C}} \otimes s \otimes \mathrm{T} \bar{C}\right) \circ\left(i \otimes \tau_{C, \mathrm{~T} \bar{C}} \otimes \mathrm{T} \bar{C}\right) \circ\left(\Delta_{C} \otimes \Delta_{\mathrm{TA} \bar{C}}\right) \circ\left(s^{-1} \otimes \mathrm{T} \bar{C}\right) .
$$

Therefore the product on $\left(\mathbb{k} \oplus s^{-1} \bar{A}\right) \otimes T \bar{A}$ is the sum of three terms: the product $\mu_{\mathrm{TC}} \bar{A}$ of $\mathrm{TC} \bar{A}$, the dual of $\Delta_{1}: s^{-1} \bar{A} \otimes \mathrm{T} \bar{A} \otimes \mathrm{T} \bar{A} \rightarrow s^{-1} \bar{A} \otimes \mathrm{T} \bar{A}$ and the dual of $\Delta_{2}: \mathrm{T} \bar{A} \otimes s^{-1} \bar{A} \otimes \mathrm{T} \bar{A} \rightarrow s^{-1} \bar{A} \otimes \mathrm{T} \bar{A}$.

Proof of Theorem 25. By Lemma 21 and Theorem 2, there is a natural DGC quasiisomorphism

$$
\mathrm{C}\left(\mathrm{TA} \overline{S_{*}(Y)}\right) \stackrel{\simeq}{\rightrightarrows} S_{*}\left((\Sigma Y)^{S^{1}}\right) .
$$

Since the cyclic bar construction (Property 8), dualizing and tensorization preserve quasi-isomorphisms between $\mathbb{k}$-free chain complexes, $\mathrm{C}\left(\mathrm{TA} \overline{S_{*}(Y)}\right)^{\vee}$ is weakly equivalent as cochain algebras to $\mathrm{C}(\mathrm{TA} \bar{C})^{\vee}$. By Lemma 23, there is a DGA quasiisomorphism

$$
\mathrm{C}(\mathrm{TA} \bar{C})^{\vee} \stackrel{\cong}{\rightrightarrows} \mathrm{C}\left(\mathbb{k} \oplus s^{-1} \overline{C^{\vee}}\right) .
$$

Therefore $\mathrm{C}\left(\mathbb{k} \oplus s^{-1} \overline{C^{\vee}}\right)$ is a weakly DGA equivalent to $S^{*}\left((\Sigma Y)^{S^{1}}\right)$.

proof of Theorem 2\%. Using Lemma 22, Theorem 11 and Lemma 23 with $C=$ $H_{*}(Y)$, we obtain the natural isomorphism of graded algebras

$$
H^{*}\left((\Sigma Y)^{S^{1}}\right) \cong H H^{*}\left(\mathrm{TA} H_{+}(Y)\right) \cong H H_{*}\left(H^{*}(\Sigma Y)\right) \text {. }
$$

Example 33. $Y=S^{d}, d \geqslant 1$. If $d \geqslant 2$ using Remark 26, $S_{*}\left(S^{d}\right)$ is weakly DGC equivalent to $\mathrm{B} \Omega H_{*}\left(S^{d}\right)$, therefore to $H_{*}\left(S^{d}\right)$. By $[\mathbf{1 7}, 7.3]$, there is a DGH quasiisomorphism $H_{*}\left(S^{1}\right) \stackrel{\simeq}{\rightarrow} S_{*}\left(S^{1}\right)$. So by Theorem 25 , as DGA

$$
S^{*}\left(\left(S^{d+1}\right)^{S^{1}}\right) \sim \mathrm{C} H^{*}\left(S^{d+1}\right)=\left(E\left(s^{-1} v\right) \otimes \mathrm{TC} v, d_{2}\right)
$$

where $v$ is an element of degree $d$. If $d$ is even in $\mathbb{k}$, as DGA

$$
S^{*}\left(\left(S^{d+1}\right)^{S^{1}}\right) \sim E\left(s^{-1} v\right) \otimes \Gamma v, 0
$$

and

$$
H^{*}\left(\left(S^{d+1}\right)^{S^{1}}\right) \cong H^{*}\left(S^{d+1}\right) \otimes H^{*}\left(\Omega S^{d+1}\right)
$$

as graded algebras. We suppose now that $d$ is odd. By dualization, $\mathrm{TC} v \cong E v \otimes$ $\mathrm{TC}\left(v^{2}\right)$ as graded algebras. So as cochain algebras

$$
S^{*}\left(\left(S^{d+1}\right)^{S^{1}}\right) \sim E\left(s^{-1} v\right) \otimes E v \otimes \Gamma\left(v^{2}\right), d_{2} \gamma_{k}\left(v^{2}\right)=2\left(s^{-1} v\right) v \gamma_{k-1}\left(v^{2}\right), k \geqslant 1 .
$$


Therefore, over any commutative ring $\mathbb{k}$, the graded algebra $H^{*}\left(\left(\Sigma S^{d}\right)^{S^{1}}\right)$ is the module

$$
\mathbb{k} \oplus\left({ }_{2} \mathbb{k}\right) \Gamma^{+}\left(v^{2}\right) \oplus \mathbb{k} v \cdot \Gamma\left(v^{2}\right) \oplus \mathbb{k}\left(s^{-1} v\right) \cdot \Gamma\left(v^{2}\right) \oplus\left(\frac{\mathbb{k}}{2 \mathbb{k}}\right) v \cdot\left(s^{-1} v\right) \cdot \Gamma\left(v^{2}\right)
$$

equipped with the obvious products. In particular, if $\frac{1}{2} \in \mathbb{k}$, all the products are trivial.

Example 34. Comparison of $Y=\mathbb{C P}^{d}$ and $Y=S^{2} \vee \cdots \vee S^{2 d}, d \geqslant 1$. The AdamsHilton model of $\mathbb{C P}^{d}$ is $\Omega H_{*}\left(\mathbb{C P}^{d}\right)$. Therefore (Remark 26$), S_{*}\left(\mathbb{C P}^{d}\right)$ is weakly DGC equivalent to $H_{*}\left(\mathbb{C P}^{d}\right)$. So by Theorem 25 , as cochain algebras

$$
S^{*}\left(\left(\Sigma \mathbb{C P}^{d}\right)^{S^{1}}\right) \sim \mathrm{C}\left(H^{*}\left(\Sigma \mathbb{C P}^{d}\right)\right) .
$$

Similarly as cochain algebras

$$
S^{*}\left(\left(S^{3} \vee \cdots \vee S^{2 d+1}\right)^{1}\right) \sim \mathrm{C}\left(H^{*}\left(S^{3} \vee \cdots \vee S^{2 d+1}\right)\right) .
$$

The cochain algebras $\mathrm{C}\left(H^{*}\left(\Sigma \mathbb{C P}^{d}\right)\right)$ and $\mathrm{C}\left(H^{*}\left(S^{3} \vee \cdots \vee S^{2 d+1}\right)\right)$ have the same underlying cochain complex. But the product on $\mathrm{C}\left(H^{*}\left(\Sigma \mathbb{C P}^{d}\right)\right)$ is the product perturbed by the algebra $H^{*}\left(\mathbb{C P}^{d}\right)$ and is far more complicated than the shuffle product on $\mathrm{C}\left(H^{*}\left(S^{3} \vee \cdots \vee S^{2 d+1}\right)\right)$.

Remark 35. Theorem 27 claims that the algebra $H^{*}\left((\Sigma Y)^{S^{1}}\right)$ depends functorially on the algebra $H^{*}(Y)$. But it is useful to remember that $H^{*}\left((\Sigma Y)^{S^{1}}\right)$ depends functorially on the Hopf algebra structure of the loop space homology $H_{*}(\Omega \Sigma Y)=$ $\mathrm{TA}_{+}(Y)$.

For example, if we return to Example 34, we obtain the weak equivalences of cochain algebras

$$
S^{*}\left(\left(\Sigma \mathbb{C P}^{d}\right)^{S^{1}}\right) \sim \mathrm{C}\left(\mathrm{TA} H_{+}\left(\mathbb{C P}^{d}\right)\right)^{\vee} \sim \mathrm{C} H^{*}\left(\Sigma \mathbb{C P}^{d}\right)
$$

and

$S^{*}\left(\left(S^{3} \vee \cdots \vee S^{2 d+1}\right)^{S^{1}}\right) \sim \mathrm{C}\left(\mathrm{TA} H_{+}\left(S^{2} \vee \cdots \vee S^{2 d}\right)\right)^{\vee} \sim \mathrm{C} H^{*}\left(S^{3} \vee \cdots \vee S^{2 d+1}\right)$.

If $\frac{1}{d !} \in \mathbb{k}$ then by a Theorem of Anick $[2,3.12]$

$$
\mathrm{TA} H_{+}\left(\mathbb{C P}^{d}\right) \cong \mathrm{TA} H_{+}\left(S^{2} \vee \cdots \vee S^{2 d}\right)
$$

as graded Hopf algebras. And so we have the isomorphism of cochain algebras

$$
\mathrm{C}\left(\mathrm{TA} H_{+}\left(\mathbb{C P}^{d}\right)\right)^{\vee} \cong \mathrm{C}\left(\mathrm{TA} H_{+}\left(S^{2} \vee \cdots \vee S^{2 d}\right)\right)^{\vee} .
$$

So finally, if $2, \ldots, d$ are units in $\mathbb{k}$, we have the isomorphism of graded Hopf algebras

$$
H^{*}\left(\Omega \Sigma \mathbb{C P}^{d}\right) \cong H^{*}\left(\Omega\left(S^{3} \vee \cdots \vee S^{2 d+1}\right)\right)
$$

and the isomorphism of graded algebras (Compare with Theorem 39 or equivalently the Theorem in $[\mathbf{1 3}]$ )

$$
H^{*}\left(\left(\Sigma \mathbb{C P}^{d}\right)^{S^{1}}\right) \cong H^{*}\left(\left(S^{3} \vee \cdots \vee S^{2 d+1}\right)^{S^{1}}\right) .
$$


The converses can be proven easily (Compare with $[\mathbf{3 4}, 9.9],[\mathbf{1 2}, \S 4]$ or $[\mathbf{3 5}$, 4.4]). Denote by $x_{2}$ the generator of $H^{2}\left(\Omega \Sigma \mathbb{C P}^{d}\right) \cong H^{2}\left(\Omega\left(S^{3} \vee \cdots \vee S^{2 d+1}\right)\right)$. In $H^{*}\left(\Omega \Sigma \mathbb{C P}^{d}\right), x_{2}^{d} \neq 0$. If $d !=0$ in $\mathbb{k}, x_{2}^{d}=0$ in $H^{*}\left(\Omega\left(S^{3} \vee \cdots \vee S^{2 d+1}\right)\right)$. Therefore, when $d !=0$ in $\mathbb{k}$, there is no isomorphism of graded algebras between $H^{*}\left(\Omega \Sigma \mathbb{C P}^{d}\right)$ and $H^{*}\left(\Omega\left(S^{3} \vee \cdots \vee S^{2 d+1}\right)\right)$. For any space $X$ such that $H_{*}(X ; \mathbb{Z})$ is $\mathbb{Z}$-free of finite type,

$$
H^{*}(X ; \mathbb{k}) \cong H^{*}(X ; \mathbb{Z}) \otimes_{\mathbb{Z}} \mathbb{k} \text { and so } H^{*}\left(X ; \frac{\mathbb{k}}{d ! \mathbb{k}}\right) \cong H^{*}(X ; \mathbb{k}) \otimes_{\mathbb{k}} \frac{\mathbb{k}}{d ! \mathbb{k}}
$$

as graded algebras. So we have the implications:

$$
\begin{aligned}
H^{*}\left(\Omega \Sigma \mathbb{C P}^{d} ; \mathbb{k}\right) & \cong H^{*}\left(\Omega\left(S^{3} \vee \cdots \vee S^{2 d+1}\right) ; \mathbb{k}\right) \text { as graded algebras } \\
\Rightarrow H^{*}\left(\Omega \Sigma \mathbb{C P}^{d} ; \frac{\mathbb{k}}{d ! \mathbb{k}}\right) & \cong H^{*}\left(\Omega\left(S^{3} \vee \cdots \vee S^{2 d+1}\right) ; \frac{\mathbb{k}}{d ! \mathbb{k}}\right) \text { as graded algebras } \\
& \Rightarrow \frac{\mathbb{k}}{d ! \mathbb{k}} \text { is the null ring } \Rightarrow \frac{1}{d !} \in \mathbb{k} .
\end{aligned}
$$

We prove now that if $d$ ! has no inverse in $\mathbb{k}$, there is no isomorphism of graded algebras between $H^{*}\left(\left(\Sigma \mathbb{C P}^{d}\right)^{S^{1}}\right)$ and $H^{*}\left(\left(S^{3} \vee \cdots \vee S^{2 d+1}\right)^{S^{1}}\right)$. We have the sequence of isomorphisms of modules $[\mathbf{2 9}, 5.3 .10]$

$H_{*}\left(\left(\Sigma \mathbb{C P}^{d}\right)^{S^{1}}\right) \cong H H_{*}\left(\mathrm{TA}\left(x_{2}, \ldots, x_{2 d}\right)\right) \cong \mathrm{T}\left(x_{2}, \ldots, x_{2 d}\right)_{\tau} \oplus(s \otimes 1) \overline{\mathrm{T}\left(x_{2}, \ldots, x_{2 d}\right)^{\tau}}$.

Since $x_{2}, \ldots, x_{2 d}$ are elements of even degree, $H_{*}\left(\left(\Sigma \mathbb{C P}^{d}\right)^{S^{1}} ; \mathbb{Z}\right)$ is $\mathbb{Z}$-free of finite type. So as for the loop spaces, the proof for the free loop spaces reduces to the case where $d !=0$ in $\mathbb{k}$. For $X=\Sigma \mathbb{C P}^{d}$ or $S^{3} \vee \cdots \vee S^{2 d+1}$, by Serre spectral sequence, the inclusion $\Omega X \hookrightarrow X^{S^{1}}$ induces in cohomology an isomorphism in degree 2 and a monomorphism in even degree. Therefore if $d !=0$ in $\mathbb{k}, x_{2}^{d} \neq 0$ in $H^{*}\left(\left(\Sigma \mathbb{C P}^{d}\right)^{S^{1}}\right)$ whereas $x_{2}^{d}=0$ in $H^{*}\left(\left(S^{3} \vee \cdots \vee S^{2 d+1}\right)^{S^{1}}\right)$.

\section{The Hochschild homology of a commutative algebra}

If a HAH model of a path connected pointed space $X$ is the cobar construction on a cocommutative chain coalgebra $C \mathbb{k}$-free of finite type such that $C=\mathbb{k} \oplus C_{\geqslant 2}$, by Theorems 11 and 18, the free loop space cohomology of $X, H^{*}\left(X^{S^{1}}\right)$, is isomorphic as graded algebras to the Hochschild homology of the CDGA $C^{\vee}$. In this section, we give various examples of such a space $X$.

Denote by $A$ the cochain algebra $C^{\vee}$. We suppose now that $A$ is strictly commutative (i. e. $a^{2}=0$ if $a \in A_{o d d}$ ) and that $\bar{A}$ is $\mathbb{k}$-semifree. We start by giving a method as general as possible to compute the Hochschild homology of $A$.

Let $V$ be a $\mathbb{k}$-free graded module of finite type. The free strictly commutative graded algebra on $V$ is denoted $\Lambda V$. The free divided powers algebra on an element $v$ has already been defined page 196. Using the formula $\Gamma(V \oplus W) \cong \Gamma V \otimes \Gamma W$ and an argument of direct limit, we define the free divided powers algebra on $V$, 
denoted $\Gamma V$. A decomposable Sullivan Model of $A$ is a cochain algebra of the form $(\Lambda V, d)$ where $V=\left\{V^{i}\right\}_{i \geqslant 2}$ is $\mathbb{k}$-free of finite type and $d(V) \subset \Lambda^{\geqslant 2} V$, equipped with a quasi-isomorphism of cochain algebras $(\Lambda V, d) \stackrel{\simeq}{\rightarrow} A$. If $\mathbb{k}$ is a principal ideal domain, by Theorem 7.1 of $[\mathbf{2 0}], A$ admits a minimal Sullivan model. When $\mathbb{k}$ is a field, minimal Sullivan models are the decomposable ones [20, Remark 7.3 i)].

Anyway, suppose now that we have somehow obtained a decomposable Sullivan model $(\Lambda V, d)$ of $A$ over our arbitrary commutative ring $\mathbb{k}$. Proposition 1.9 of [15] (See also $[\mathbf{2 1}, \mathrm{p} 320-2]$ ) is valid over any commutative ring $\mathbb{k}$, since it is proved " by induction on a basis of $V$ ordered by increasing degree ". Therefore consider the multiplication of $(\Lambda V, d)$ :

$$
\mu:\left(\Lambda V^{\prime}, d\right) \otimes\left(\Lambda V^{\prime \prime}, d\right) \rightarrow(\Lambda V, d) .
$$

By induction on the degree of $V$, we can construct a factorization of $\mu$ :

$$
\left(\Lambda V^{\prime}, d\right) \otimes\left(\Lambda V^{\prime \prime}, d\right) \stackrel{i}{\longmapsto}\left(\Lambda V^{\prime} \otimes \Lambda V^{\prime \prime} \otimes \Gamma s V, D\right) \underset{\phi}{\stackrel{\simeq}{\rightarrow}}(\Lambda V, d)
$$

such that

(i) $D(s v)-\left(v^{\prime}-v^{\prime \prime}\right) \in \Lambda\left(V^{<n}\right) \otimes \Lambda\left(V^{<n}\right) \otimes \Gamma s\left(V^{<n}\right)$ for $v \in V^{n}$,

(ii) $D\left(\gamma^{k}(s v)=D(s v) \gamma^{k-1}(s v)\right.$ for $v \in V^{o d d}$ and

(iii) $\phi\left(\Gamma(s V)^{+}\right)=0$.

Moreover, any such factorization satisfies

(iv) $i$ is an inclusion of CDGA's such that $\left(\Lambda V^{\prime} \otimes \Lambda V^{\prime \prime} \otimes \Gamma s V, D\right)$ is $\left(\Lambda V^{\prime}, d\right) \otimes$ $\left(\Lambda V^{\prime \prime}, d\right)$-semifree,

(v) $\phi$ is a CDGA quasi-isomorphism and

(vi) $\operatorname{Im} D \subset\left(\Lambda V^{\prime} \otimes \Lambda V^{\prime \prime}\right)^{+} \otimes \Gamma s V$.

By push out in the category of CDGA's, the multiplication of $A$ extends to a CDGA quasi-isomorphism from

$$
(A \otimes A) \otimes_{(\Lambda V, d) \otimes(\Lambda V, d)}\left(\Lambda V^{\prime} \otimes \Lambda V^{\prime \prime} \otimes \Gamma s V, D\right),
$$

which is $A \otimes A$-semifree, to $A$. The multiplication of $A$ also extends to a CDGA quasiisomorphism $\mathrm{B}(A ; A ; A) \stackrel{\cong}{\rightrightarrows} A$. Since $\bar{A}$ is $\mathbb{k}$-semifree, the bar resolution $\mathrm{B}(A ; A ; A)$ is also $A \otimes A$-semifree. Therefore the cochain algebras

$$
\begin{aligned}
\mathrm{C}(A) & =A \otimes_{A \otimes A} \mathrm{~B}(A ; A ; A), \\
(A \otimes \Gamma s V, \bar{D}) & =A \otimes_{A \otimes A}(A \otimes A) \otimes_{(\Lambda V, d) \otimes(\Lambda V, d)}\left(\Lambda V^{\prime} \otimes \Lambda V^{\prime \prime} \otimes \Gamma s V, D\right)
\end{aligned}
$$

and

$$
(\Lambda V \otimes \Gamma s V, \bar{D})=(\Lambda V, d) \otimes_{(\Lambda V, d) \otimes(\Lambda V, d)}\left(\Lambda V^{\prime} \otimes \Lambda V^{\prime \prime} \otimes \Gamma s V, D\right)
$$

are weakly equivalent as CDGA's ([30, VIII.2.3], for details [34, Section 8] or [3, 2.3.2]). So finally, we have the isomorphisms of graded algebras

$$
H H_{*}(A) \cong H^{*}(A \otimes \Gamma s V, \bar{D}) \cong H^{*}(\Lambda V \otimes \Gamma s V, \bar{D}) .
$$


Proposition 36. The free loop space cohomology on the complex projective space $\mathbb{C P}^{n}, H^{*}\left(\left(\mathbb{C P}^{n}\right)^{S^{1}}\right)$, is isomorphic as graded algebra to the Hochschild homology of $H^{*}\left(\mathbb{C P}^{n}\right)$.

The same result (same proof) holds for the quaternionic projective space $\mathbb{H}^{n}$.

Lemma 37. [2, 8.1g),h)][1, Corollary 2.1] [34, part 1. of Theorem 6.2] Let $X \hookrightarrow$ $Y$ be an inclusion of simply-connected $C W$-complexes. Consider an Adams-Hilton model of $X$, equipped with a HAH model structure for $X, \mathcal{A}(X)$. Consider an Adams-Hilton model of $Y, \mathcal{A}(Y)$ extending the Adams-Hilton model of $X$. Then there is an structure of $H A H$ model for $Y$ on $\mathcal{A}(Y)$ that extends the HAH model structure for $X$ of $\mathcal{A}(X)$.

Proof of Proposition 36. By induction on $n$, we suppose that the Adams-Hilton model of $\mathbb{C P}^{n-1}$ equipped with its HAH model structure is the cobar construction $\Omega H_{*}\left(\mathbb{C P}^{n-1}\right)$ equipped with the shuffle diagonal, denoted $\Delta_{s}$. Denote by $\Delta$ the diagonal on $\Omega H_{*}\left(\mathbb{C P}^{n}\right)=\mathrm{TA}\left(z_{1}, \ldots, z_{2 n-1}, d_{2}\right)$ obtained by Lemma 37 . This diagonal $\Delta$ is different from the shuffle diagonal $\Delta_{s}$ only on the top generator $z_{2 n-1}$. So $\left(\Delta_{s}-\Delta\right) z_{2 n-1}$ is a cycle. Since $\Omega \mathbb{C P}^{n} \approx S^{1} \times \Omega S^{2 n-1}$, for degree reason, it is a boundary. Therefore, we can construct a derivation homotopy from $\Delta_{s}$ to $\Delta$.

A decomposable Sullivan model of $\frac{\mathbb{k}\left[x_{2}\right]}{x_{2}^{n+1}}$ is $\left(\Lambda\left(x_{2}, y_{2 n+1}\right), d\right)$ with $d y_{2 n+1}=x_{2}^{n+1}$. Using the general method described above, $H H_{*}\left(H^{*}\left(\mathbb{C P}^{n}\right)\right)$ is the cohomology algebra of

$$
\left(\frac{\mathbb{k}\left[x_{2}\right]}{x_{2}^{n+1}} \otimes \Gamma s x_{2}, s y_{2 n+1}, \bar{D}\right)
$$

with $\bar{D} s y_{2 n+1}=s x(n+1) x^{n}$. Therefore the graded algebra $H^{*}\left(\left(\mathbb{C P}^{n}\right)^{S^{1}}\right)$ is the module

$$
\begin{array}{r}
\mathbb{k} \oplus \bigoplus_{1 \leqslant p \leqslant n, i \in \mathbb{N}} \mathbb{k} x^{p} \gamma^{i}(s y) \oplus \bigoplus_{0 \leqslant p \leqslant n-1, i \in \mathbb{N}} \mathbb{k} x^{p} s x \gamma^{i}(s y) \\
\oplus \bigoplus_{i \in \mathbb{N}} \frac{\mathbb{k}}{(n+1) \mathbb{k}} x^{n} s x \gamma^{i}(s y) \oplus \bigoplus_{i \in \mathbb{N}^{*}}\left({ }_{n+1} \mathbb{k}\right) \gamma^{i}(s y)
\end{array}
$$

equipped with the obvious products. When $\mathbb{k}=\mathbb{Z}$, this is exactly Proposition 15.33 of $[\mathbf{1 0}]$ (Set $\alpha_{i}=x \gamma^{i}(s y)$ and $\beta_{i}=s x \gamma^{i}(s y)$ to make the correspondence). In particular, if $n+1=0$ in $\mathbb{k}$, we obtain the isomorphism of graded algebras

$$
H^{*}\left(\left(\mathbb{C P}^{n}\right)^{S^{1}}\right) \cong H^{*}\left(\mathbb{C P}^{n}\right) \otimes H^{*}\left(\left(\Omega \mathbb{C P}^{n}\right)\right) .
$$

To compute the Hochschild cohomology of a universal enveloping algebra of a Lie algebra is equivalent to computing the Hochschild homology of a commutative algebra:

Consider a differential graded Lie algebra (in the sense of $[\mathbf{2 0}, 1.1(i)]) L$ such that $L=\left\{L_{i}\right\}_{i \geqslant 1}$ is $\mathbb{k}$-free of finite type. The universal envelopping algebra of $L$, denoted $U L$, has a natural structure of DGH [20,1.1(ii)]. If $\frac{1}{2} \in \mathbb{k}$, the reduced bar construction $\mathrm{B}(U L)$ contains a quasi-isomorphic sub-DGC $\mathcal{C}_{*}(L)=\left(\Gamma s L, d_{1}+\right.$ 
$\left.d_{2}\right)$. Its dual, denoted $\mathcal{C}^{*}(L)$, is a CDGA called the (reduced) Cartan-ChevalleyEilenberg complex. Since $\mathcal{C}_{*}(L)$ is cocommutative, the cobar construction $\Omega \mathcal{C}_{*}(L)$ equipped with the shuffle diagonal is a DGH. The composite of natural DGA quasiisomorphisms

$$
\Omega \mathcal{C}_{*}(L) \stackrel{\simeq}{\rightarrow} \Omega \mathrm{B}(U L) \stackrel{\simeq}{\rightrightarrows} U L
$$

is a DGH morphism. By Theorem 18, we get immediately

Lemma 38. Suppose that $\frac{1}{2} \in \mathbb{k}$. Let $L$ be a differential graded Lie algebra such that $L=\left\{L_{i}\right\}_{i \geqslant 1}$ is $\mathbb{k}$-free of finite type. Then there is a natural isomorphism of commutative graded algebras

$$
H H^{*}(U L) \cong H H_{*}\left(\mathcal{C}^{*}(L)\right) .
$$

We give now a large class of spaces which admit the universal enveloping algebra of a differential graded Lie algebra as a HAH model:

Let $r \geqslant 1$ be a fixed integer. Let $p \geqslant 2$ be an integer (possibly infinite) such that $\frac{1}{(p-1) !} \in \mathbb{k}$. Consider a $r$-connected $\mathrm{CW}$-complex $X$ of finite type and of dimension $\leqslant r p$. We want to compute the free loop space cohomology algebra $H^{*}\left(X^{S^{1}}\right)$. If $p=2$ then, by the Freudenthal Suspension Theorem and Proposition 27.5 of [18], $X$ is the suspension of a co-H space. And so we have already seen in Section 7, particularly using Corollary 28, how to compute its free loop space cohomology algebra $H^{*}\left(X^{S^{1}}\right)$. Therefore, we can suppose that $p \neq 2$. The Adams-Hilton model of $X, \mathcal{A}(X)$, is a chain algebra of the form (TA $V, d)$ on a $\mathbb{k}$-free graded module $V$ concentrated in degrees between $r$ and $r p-1$, endowed with a structure of $\mathrm{HAH}$ model for $X$. Therefore, by a deep Theorem of Anick $[\mathbf{2}, 5.6]$, there exists a free graded submodule $W \subset \mathrm{TA} V$ such that $d(W)$ embeds into the free graded Lie algebra generated by $W, \mathbb{L} W \subset \mathrm{TA} V$ and such that the DGA morphism

$$
U(\mathbb{L} W, d) \stackrel{\cong}{\longrightarrow}(\mathrm{TA} V, d)
$$

is a HAH isomorphism. This free differential graded Lie algebra $(\mathbb{L} W, d)$ is the model $\mathbf{L}(X)$ of Construction 8.4 of [2]. By Lemma 38, we have

Theorem 39. With the above hypothesis and notations, there is a natural isomorphism of graded algebras

$$
H^{*}\left(X^{S^{1}}\right) \cong H H_{*}\left(\mathcal{C}^{*}(\mathbf{L}(X))\right) .
$$

This Theorem extends the rational case given by Vigué and Sullivan [41]. When $\mathbb{k}$ is a field, this Theorem has been first proved by Dupont and Hess [13]. We had the idea of studying free loop space for "large primes" after studying fibrations for "large primes" in [34].

\section{References}

[1] J. F. Adams and P. J. Hilton, On the chain algebra of a loop space, Comment. Math. Helv. 30 (1955), 305-330. 
[2] D. J. Anick, Hopf algebras up to homotopy, J. Amer. Math. Soc. 2 (1989), no. $3,417-453$.

[3] L. Avramov, Infinite free resolutions, Six lectures on commutative algebra. (Bellaterra, 1996), Prog. Math., vol. 166, Birkhäuser, Basel, 1998, pp. 1-118.

[4] H. J. Baues, Algebraic homotopy, Cambridge Univ. Press, 1989.

[5] R. Bott, Lectures on Gelfand-Fuks cohomology and characteristic classes. Notes by M. Mostow and J. Perchik, New Mexico State University 1973, Raoul Bott: Collected Papers. Vol. 3. Foliations (R. D. Mac Pherson, ed.), Contemporary Mathematicians, Birkhäuser Boston, 1995.

[6] A. K. Bousfield and D. M. Kan, Homotopy limits, completions and localizations, Lectures Notes in Mathematics, vol. 304, Springer-Verlag, Berlin-New York, 1972.

[7] D. Burghelea and Z. Fiedorowicz, Cyclic homology and algebraic K-theory of spaces. II, Topology 25 (1986), no. 3, 303-317.

[8] H. Cartan and Eilenberg S., Homological algebra, Princeton University Press, 1956.

[9] R. Cohen, A model for the free loop space of a suspension, Algebraic topology (Seattle, Wash., 1985), Lecture Notes in Math., vol. 1286, Springer, BerlinNew York, 1987, pp. 193-207.

[10] M. Crabb and I. James, Fibrewise homotopy theory, Springer Monographs in Mathematics, Springer-Verlag, London, 1998.

[11] A. Dold, Lectures on algebraic topology, reprint of the 1972 ed., Classics in Mathematics, Springer-Verlag, Berlin, 1995.

[12] N. Dupont and K. Hess, Commutative free loop space models at large primes, Math. Z., to be published.

[13] - Hochschild cohomology is topological, J. Pure Appl. Algebra, to be published.

[14] How to model the free loop space algebraically, Math. Ann. 314 (1999), no. 3, 469-490.

[15] Y. Félix, S. Halperin, C. Jacobsson, C. Löfwall, and J.-C. Thomas, The radical of the homotopy lie algebra, Amer. J. Math. 110 (1988), no. 2, 301-322.

[16] Y. Félix, S. Halperin, and J.-C. Thomas, Adam's cobar equivalence, Trans. Amer. Math. Soc. 329 (1992), 531-549.

[17] Differential graded algebras in topology, Handbook of Algebraic Topology (I. M. James, ed.), North-Holland, Amsterdam, 1995, pp. 829-865.

[18] - Rational homotopy theory, Graduate Texts in Mathematics, vol. 205, Springer-Verlag, 2000.

[19] T. G. Goodwillie, Cyclic homology, derivations, and the free loopspace, Topology 24 (1985), no. 2, 187-215.

[20] S. Halperin, Universal enveloping algebras and loop space homology, J. Pure Appl. Algebra 83 (1992), 237-282. 
[21] S. Halperin and M. Vigué-Poirrier, The homology of a free loop space, Pacific J. Math. 147 (1991), no. 2, 311-324.

[22] K. Hess, Perturbation and transfer of generic algebraic structure, Higher homotopy structures in topology and mathematical physics (Poughkeepsie, NY, 1996), Contemp. Math., vol. 227, Amer. Math. Soc., 1999, pp. 103-143.

[23] D. Husemoller, Fibre bundles, third ed., Graduate Texts in Mathematics, no. 20, Springer-Verlag, New York, 1994.

[24] E. Idrissi, L'isomorphisme de Jones-McCleary et celui de Goodwillie sont des isomorphismes d'algèbres, C. R. Acad. Sci. Paris Sér. I Math. 331 (2000), $507-510$.

[25] J. D. S. Jones, Cyclic homology and equivariant homology, Invent. Math. 87 (1987), no. 2, 403-423.

[26] J. D. S. Jones and J. McCleary, Hochschild homology, cyclic homology, and the cobar construction, Adams Memorial Symposium on Algebraic Topology, 1 (Manchester, 1990), London Math. Soc. Lecture Note Ser., vol. 175, Cambridge Univ. Press, 1992, pp. 53-65.

[27] K. Kuribayashi and T. Yamaguchi, The cohomology algebra of certain free loop spaces, Fund. Math. 154 (1997), no. 1, 57-73.

[28] L. A. Lambe, Homological perturbation theory, Hochschild homology, and formal groups, Deformation theory and quantum groups with applications to mathematical physics (Amherst, MA, 1990), Contemp. Math., vol. 134, Amer. Math. Soc., 1992, pp. 138-218.

[29] J. Loday, Cyclic homology, second ed., Grundlehren der Mathematischen Wissenschaften, vol. 301, Springer-Verlag, Berlin, 1998.

[30] S. Mac Lane, Homology, Springer-Verlag, Berlin, 1963.

[31] - Categories for the working mathematician, Graduate Texts in Mathematics, vol. 5, Springer-Verlag, New York-Berlin, 1971.

[32] J. P. May, The cohomology of restricted Lie algebras and of Hopf algebras, J. Algebra 3 (1966), 123-146.

[33] - The geometry of iterated loop spaces, Lectures Notes in Mathematics, vol. 271, Springer-Verlag, Berlin-New York, 1972.

[34] L. Menichi, On the cohomology algebra of a fiber, Preprint Hopf Topology Archive, September 1999.

[35] Bitjong Ndombol and J.-C. Thomas, On the cohomology algebra of free loop spaces, Prépublications, Université d'Angers, 1999, http://math.univangers.fr/preprint.html, to appear in Topology.

[36] M. Parhizgar, On the cohomology ring of the free loop space of a wedge of spheres, Math. Scand. 80 (1997), no. 2, 195-248.

[37] G. Segal, Categories and cohomology theories, Topology 13 (1974), 293-312.

[38] A. Solotar, Cyclic homology of a free loop space, Comm. Algebra 21 (1993), no. $2,575-582$. 
[39] D. Tanré, Homotopie rationnelle: Modèles de Chen, Quillen, Sullivan, Lecture Notes in Mathematics, vol. 1025, Springer-Verlag, Berlin-New York, 1983.

[40] M. Vigué-Poirrier, Homologie de hochschild et homologie cyclique des algbres différentielles graduées, International Conference on Homotopy Theory (Marseille-Luminy, 1988), Astérisque, no. 191, 1990, pp. 255-267.

[41] M. Vigué-Poirrier and D. Sullivan, The homology theory of the closed geodesic problem, J. Differential Geometry 11 (1976), no. 4, 633-644.

This article may be accessed via WWW at http://www.rmi.acnet.ge/hha/ or by anonymous ftp at

ftp://ftp.rmi.acnet.ge/pub/hha/volumes/2001/n9/n9.(dvi,ps,dvi.gz,ps.gz)

Luc Menichi Luc.Menichi@univ-angers.fr

Université d'Angers, Faculté des Sciences

2 Boulevard Lavoisier

49045 Angers, FRANCE 